\title{
Boundary amenability of hyperbolic spaces
}

\author{
Vadim A. Kaimanovich
}

\begin{abstract}
It is well-known that a Kleinian group is amenable if and only if it is elementary. We establish an analogous property for equivalence relations and foliations with Gromov hyperbolic leaves: they are amenable if and only if they are elementary in the sense that one can assign (in a measurable way) to any leaf a finite subset of its hyperbolic boundary (as in the group case, such subsets cannot actually contain more than 2 points). The analogous result for actions of word hyperbolic groups with a quasi-invariant measure is that such an action is amenable if and only if it factorizes through the hyperbolic boundary or its symmetric square.

A byproduct of our approach is a proof of boundary amenability for isometry groups of Gromov hyperbolic spaces under the assumption that either the space is exponentially bounded or it is CAT $(-1)$ and the group has a finite critical exponent. We also give examples showing that without these assumptions the boundary amenability may fail.
\end{abstract}

\section{Introduction}

The class of amenable groups is, from the analytical point of view, the most natural generalization of the class of compact groups. Amenable groups are those which admit an invariant mean (rather than an invariant probability measure, which is the case for compact groups). There are many different equivalent definitions of amenability. Probably, the most constructive definition (used for verifying amenability of a given group) is the one formulated in terms of existence of approximatively invariant sequences of probability measures on the group (Reiter's condition), whereas one of the main applications of amenability is the fixed point property for affine actions of amenable groups on compact spaces.

2000 Mathematics Subject Classification. Primary 37A20, 43A07, 53C99; Secondary 20F67, $28 \mathrm{~A} 20,53 \mathrm{C} 12$.

Key words and phrases. Amenability, hyperbolic space, boundary, equivalence relation, measurability, foliation, transversal.

The paper was mostly written during author's stay at the Max-Planck-Institut (Bonn) in 2002. Its financial support and the excellent working conditions are gratefully acknowledged. I would also like to thank the organizers of the First JAMS Symposium "Discrete Geometric Analysis", where these results were presented, and, in particular, Professor Motoko Kotani, for creating an ideal atmosphere at this very fruitful meeting. I am grateful to Theo Bühler and to the anonymous referee for a number of useful comments and suggestions.

(C)0000 (copyright holder) 
It turns out that non-amenable groups may still have actions which look like actions of amenable groups. This observation led Zimmer [Zim77, Zim78 to introduce the notion of an amenable action. In the same way as with groups, there are several definitions of an amenable action. In particular, amenable actions can be characterized both in terms of a fixed point property (this was the original definition of Zimmer) and in terms of existence of a sequence of approximatively equivariant maps from the action space to the space of probability measures on the group (this is an analogue of Reiter's condition). Yet another generalization is the notion of amenability for equivalence relations and foliations. Actually, all these objects can be considered as measured groupoids, and the notion of amenability in each particular case is a specialization of a general notion of an amenable groupoid, see ADR00, CHLI02.

It is well-known that a Kleinian group is amenable if and only if it is elementary. We establish an analogous property for equivalence relations and foliations with Gromov hyperbolic leaves: under a suitable bounded geometry condition they are amenable if and only if they are elementary in the sense that one can assign (in a measurable way) to any leaf a finite subset of its hyperbolic boundary (actually, such subsets cannot consist of more than 2 points). As particular cases it contains and generalizes earlier results on amenability of boundary actions of isometry groups of hyperbolic spaces and on amenability of the stable foliation of the geodesic flow.

The main geometric ingredients of our approach - (i) boundary convergence of geodesics in hyperbolic spaces (in various guises), and (ii) the notion of the barycenter for hyperbolic spaces - are well-known and have been used before (e.g., see Ada96, BM96). However, working with hyperbolic equivalence relations requires developing a new technique (based on the notion of a measurable bundle of Banach spaces) which allows one to deal with measurable systems of (possibly different) leafwise hyperbolic boundaries. Another technical novelty in this paper is the use of simple explicit constructions of approximatively invariant sequences of measures as Cesaro averages for sandwiched sequences of sets with subexponential growth (Theorem 1.33 and Lemma 1.35) and Cesaro averages of pre-Patterson measures along geodesics (Theorem 1.38).

The paper has the following structure. In Section 1 we collect the geometric properties of Gromov hyperbolic spaces used in the sequel. These are Proposition 1.3 on boundary convergence of geodesic rays, Proposition 1.18 on existence of (quasi)-barycenter sets and Theorems 1.33 1.38 on existence of approximatively invariant boundary maps.

The main technical tool used to ensure measurability of leafwise application of these constructions in the setup of graphed equivalence relations with hyperbolic leaves is the measurable Banach bundle of leafwise functions with a continuous boundary extension (Section 2.1). By using this bundle we define measurable systems of boundary measures and show that a hyperbolic equivalence relation admits an invariant boundary system of probability measures if and only if it is elementary (Theorem 2.22). Further we establish an analogous result for measurable foliations (Theorem 2.31) by reducing them to equivalence relations.

We begin Section 3 with illustrating the key geometric ingredients of the relationship between amenability and hyperbolicity, namely, boundary convergence of geodesics and existence of quasi-barycenter sets, on the example of coincidence of the class of amenable groups of isometries of Gromov hyperbolic spaces with 
the class of elementary groups (under suitable bounded geometry type conditions). Further we proceed to establish analogues of this result for equivalence relations, foliations and actions (Theorem 3.4 Theorem 3.9 and Theorem 3.14 respectively) by taking stock of the results from the previous sections and following precisely the same scheme: amenability by the fixed point property implies existence of an invariant system of boundary measures, and therefore elementarity, whereas elementarity leads to existence of approximatively invariant sequences of probability measures, i.e., to amenability. As a byproduct we give a proof of the topological amenability of the boundary action of the group of isometries of a hyperbolic space under the assumption that either the space is exponentially bounded or the space is CAT $(-1)$ and the group has a finite critical exponent (Theorem 3.15] and Theorem 3.17). Finally, we give examples showing that without these assumptions the boundary amenability (even in the weakest form) may well fail.

\section{Asymptotic geometry of hyperbolic spaces}

1.1. Hyperbolic spaces. A detailed discussion of the notion of $\delta$-hyperbolicity and of the associated structures can be found in the seminal work of Gromov Gro87 and in the notes GdIH90. Below are listed some of the definitions and properties used later on.

We shall choose the definition based on the Rips condition: a non-compact complete proper geodesic metric space $\mathcal{X}$ is $\delta$-hyperbolic (with $\delta \geq 0$ ) if each of the sides of any geodesic triangle is contained in the $\delta$-neighbourhood of the union of the other two sides (see, for instance, GdlH90 Proposition 2.21] for a list of other equivalent definitions). The minimal number $\delta$ with this property is the hyperbolicity constant of $\mathcal{X}$. A graph is called $\delta$-hyperbolic if the associated 1complex with length 1 edges is $\delta$-hyperbolic. Usually, we shall not be concerned with the precise value of the hyperbolicity constant, and call the above spaces just hyperbolic.

For the rest of this Section we shall fix a hyperbolic space $\mathcal{X}$ with metric $d$ and the hyperbolicity constant $\delta$. Denote by

$$
(y \mid z)_{x}=\frac{1}{2}[d(x, y)+d(x, z)-d(y, z)], \quad x, y, z \in \mathcal{X}
$$

the Gromov product on $\mathcal{X}$. Then by GdlH90 Lemme 2.17 and Proposition 2.21] for any geodesic segment $[y, z]$ joining the points $y$ and $z$

$$
d(x,[y, z])-4 \delta \leq(y \mid z)_{x} \leq d(x,[y, z]) .
$$

The functions on $\mathcal{X} \times \mathcal{X}$

$$
\varrho_{x}(y, z)= \begin{cases}0, & y=z, \\ e^{-(y \mid z)_{x}}, & \text { otherwise },\end{cases}
$$

determine a uniform structure "at infinity" on the space $\mathcal{X}$ (which does not depend on the choice of the point $x$ ). The completion $\overline{\mathcal{X}}$ of $\mathcal{X}$ with respect to this uniform structure is called the hyperbolic compactification of $\mathcal{X}$. Its boundary is denoted by $\partial \mathcal{X}=\overline{\mathcal{X}} \backslash \mathcal{X}$. The action of the group of isometries $\operatorname{Iso}(\mathcal{X})$ extends from $\mathcal{X}$ to a continuous action on $\partial \mathcal{X}$.

Any geodesic ray $\xi$ converges in the hyperbolic compactification to a boundary point $\xi(\infty) \in \partial \mathcal{X}$. The definition of $\delta$-hyperbolicity is easily seen to imply 
the following property (which is, for instance, essentially contained in GdlH90, Proposition 7.2]):

Proposition 1.3. There exists a constant $C_{1}=C_{1}(\delta)$ with the property that for any $\delta$-hyperbolic space $\mathcal{X}$ and any two geodesic rays $\xi_{1}, \xi_{2}$ in $\mathcal{X}$ with $\xi_{1}(\infty)=\xi_{2}(\infty)$ there is a number $T=T\left(\xi_{1}, \xi_{2}\right)$ such that $|T| \leq d\left(\xi_{1}(0), \xi_{2}(0)\right)$ and

$$
d\left(\xi_{1}(t), \xi_{2}(t+T)\right) \leq C_{1} \quad \forall t \geq d\left(\xi_{1}(0), \xi_{2}(0)\right) .
$$

Although the functions $\varrho_{x}$ (1.2) do not, in general, satisfy the triangle inequality, they are "almost" metrics. Namely, for $a=\frac{1}{15 \delta}$ the inner metric

$$
\rho_{x}(y, z)=\inf \left\{\sum_{i=1}^{n}\left[\varrho_{x}\left(x_{i-1}, x_{i}\right)\right]^{a}: n \geq 1, y=x_{0}, x_{1}, \ldots, x_{n}=z \in \mathcal{X}\right\}
$$

determined by the function $\varrho_{x}^{a}$ satisfies the inequality

$$
\frac{1}{2}\left(\varrho_{x}\right)^{a} \leq \rho_{x} \leq\left(\varrho_{x}\right)^{a}
$$

(this is an adaptation of Woe00 Proposition 22.8]). So, the hyperbolic compactification $\overline{\mathcal{X}}$ is the completion of $\mathcal{X}$ with respect to any of the metrics $\rho_{x}$.

Notation. Below we shall use the notation $B(o, r)$ (resp., $S(o, r)$ ) for the $r$ ball (resp., the $r$-sphere) in $\mathcal{X}$ of the hyperbolic metric $d$ around a point $o \in \mathcal{X}$, and $B_{x}(o, r)$ (resp., $S_{x}(o, r)$ ) for the $r$-ball (resp., the $r$-sphere) in $\overline{\mathcal{X}}$ of the metric $\rho_{x}$ around a point $o \in \overline{\mathcal{X}}$. We denote the space of probability measures on a topological space $X$ by $\mathcal{P}(X)$, and the normalized restriction of a measure $m$ to a positive measure set $A$ by $m_{A}$.

\subsection{The barycenter set of a boundary measure. Denote by}

$$
\beta_{z}(x, y)=d(y, z)-d(x, z)=d(x, y)-2(y \mid z)_{x} \quad x, y \in \mathcal{X}
$$

the distance cocycle associated with a point $z \in \mathcal{X}$. The triangle inequality for the metric $d$ and formula (1.5) imply

Proposition 1.7. For any $x, y, z \in \mathcal{X}$ the distance cocycle satisfies the inequalities

and (assuming $y \neq z$ )

$$
\left|\beta_{z}(x, y)\right| \leq d(x, y)
$$

$$
d(x, y)+\frac{2}{a} \log \rho_{x}(y, z) \leq \beta_{z}(x, y) \leq d(x, y)+\frac{2}{a} \log \rho_{x}(y, z)+C_{2},
$$

where

$$
C_{2}=C_{2}(\delta)=\frac{2 \log 2}{a}=(30 \log 2) \delta .
$$

Corollary 1.8. For any $\gamma \in \partial \mathcal{X}$ and $x, y \in \mathcal{X}$

$$
\limsup _{z \rightarrow \gamma} \beta_{z}(x, y)-\liminf _{z \rightarrow \gamma} \beta_{z}(x, y) \leq C_{2} \text {. }
$$

Put

$$
\bar{\beta}_{\gamma}(x, y)=\limsup _{z \rightarrow \gamma} \beta_{z}(x, y) \quad x, y \in \mathcal{X}, \gamma \in \partial \mathcal{X} .
$$

As it follows from (1.6), the functions $\bar{\beta}_{\gamma}$ are Lipschitz with respect to each of the arguments. Although $\bar{\beta}_{\gamma}$ are not, generally speaking, cocycles, they still satisfy 
the cocycle identity with a uniformly bounded error (i.e., they are quasi-cocycles). More precisely, Proposition 1.7 and Corollary 1.8 imply

Proposition 1.9. For any $\gamma \in \partial \mathcal{X}$ the function $\bar{\beta}_{\gamma}$ has the following properties:

(i) $\bar{\beta}_{\gamma}$ is "jointly Lipschitz", i.e., $\left|\bar{\beta}_{\gamma}(x, y)\right| \leq d(x, y)$ for all $x, y \in \mathcal{X}$, in particular, $\bar{\beta}_{\gamma}(x, x) \equiv 0$;

(ii) $0 \leq \bar{\beta}_{\gamma}(x, y)+\bar{\beta}_{\gamma}(y, x) \leq C_{2}$ for all $x, y \in \mathcal{X}$;

(iii) $0 \leq \bar{\beta}_{\gamma}(x, y)+\bar{\beta}_{\gamma}(y, z)+\bar{\beta}_{\gamma}(z, x) \leq 2 C_{2}$ for all $x, y, z \in \mathcal{X}$.

REMARK 1.10. Our definition of $\bar{\beta}_{\gamma}$ is different from the definition of the "Busemann function" in GdlH90 Chapitre 8] (where the point $z$ was allowed to converge to $\gamma$ along geodesic rays only).

For any probability measure $\lambda \in \mathcal{P}(\partial \mathcal{X})$ put

$$
\mathcal{B}_{\lambda}(x, y)=\int \bar{\beta}_{\gamma}(x, y) d \lambda(\gamma) .
$$

The Lipschitz property of the functions $\beta_{\gamma}$ implies that $\mathcal{B}_{\lambda}$ are also Lipschitz with respect to each of the arguments. Moreover, as it follows from Proposition 1.9] the functions $\mathcal{B}_{\lambda}$ are jointly Lipschitz, and they are quasi-cocycles with the same constants as in Proposition [1.9] In particular,

$$
\left|\mathcal{B}_{\lambda}(x, y)-\mathcal{B}_{\lambda}\left(x^{\prime}, y\right)-\mathcal{B}_{\lambda}\left(x, x^{\prime}\right)\right| \leq 3 C_{2} \quad \forall x, x^{\prime}, y \in \mathcal{X}, \lambda \in \mathcal{P}(\partial \mathcal{X}) .
$$

Proposition 1.12. If a sequence of probability measures $\lambda_{n} \in \mathcal{P}(\mathcal{X})$ is convergent in the weak ${ }^{*}$ topology to a measure $\lambda \in \mathcal{P}(\mathcal{X})$, then

$$
\left|\mathcal{B}_{\lambda}(x, y)-\limsup _{n \rightarrow \infty} \int \beta_{z}(x, y) d \lambda_{n}(z)\right| \leq C_{2} \quad \forall x, y \in \mathcal{X} .
$$

Proof. As it follows from Proposition 1.7 if $x \neq y$ then both terms in the left-hand side belong to the interval

$$
\left[d(x, y)+\frac{2}{a} \int \log \rho_{x}(y, \gamma) d \lambda(\gamma), d(x, y)+\frac{2}{a} \int \log \rho_{x}(y, \gamma) d \lambda(\gamma)+C_{2}\right] .
$$

Proposition 1.13. If a measure $\lambda \in \mathcal{P}(\partial \mathcal{X})$ does not have atoms of weight at least $\frac{1}{2}$, then for any $x \in X$

$$
\lim _{y} \mathcal{B}_{\lambda}(x, y)=\infty
$$

if $y \rightarrow \infty$ in the space $\mathcal{X}$ (i.e., $d(x, y) \rightarrow \infty$ ).

Proof. Without loss of generality we may assume that $y \rightarrow \gamma_{0} \in \partial \mathcal{X}$. Choose $\varepsilon>0$ in such a way that $\lambda B_{x}\left(\gamma_{0}, \varepsilon\right)<\frac{1}{2}$, and, by using the inequalities from Proposition 1.7 (which are satisfied for the functions $\bar{\beta}_{\gamma}$ as well), estimate $\bar{\beta}_{\gamma}(x, y)$ from below as

$$
\bar{\beta}_{\gamma}(x, y) \geq \begin{cases}-d(x, y), & \gamma \in B_{x}\left(\gamma_{0}, \varepsilon\right), \\ d(x, y)+\frac{2}{a} \log \rho_{x}(y, \gamma), & \gamma \in \partial \mathcal{X} \backslash B_{x}\left(\gamma_{0}, \varepsilon\right) .\end{cases}
$$

Since $y \rightarrow \gamma_{0}$, we may assume that $\rho_{x}(y, \gamma) \geq \varepsilon / 2$ for any $\gamma \in \partial \mathcal{X} \backslash B_{x}\left(\gamma_{0}, \varepsilon\right)$, whence integrating the above estimate yields the claim. 
REMARK 1.14. If $\mathcal{X}$ is a $\operatorname{CAT}(-1)$ space, then all functions $\bar{\beta}_{\gamma}$ and $\mathcal{B}_{\lambda}$ are actually cocycles, so that under conditions of Proposition 1.13 the function $\mathcal{B}_{\lambda}(x, \cdot)$ attains its minimum on a compact subset of $\mathcal{X}$ (the barycenter set of $\lambda$ ) which does not depend on the choice of the point $x \in \mathcal{X}$, see DE86, BCG96, BM96. Due to the usual technical complications connected with replacing the CAT $(-1)$ property with $\delta$-hyperbolicity, this is no longer true for a general $\delta$-hyperbolic space, which is why we have to "quasify" the definition of the barycenter set.

Definition 1.15. Given a number $r>0$ the set

$$
\mathcal{C}(\lambda, x, r)=\left\{y \in \mathcal{X}: \mathcal{B}_{\lambda}(x, y) \leq r+\inf _{z \in \mathcal{X}} \mathcal{B}_{\lambda}(x, z)\right\}
$$

is called the quasi-barycenter set of the measure $\lambda \in \mathcal{P}(\partial \mathcal{X})$ with respect to the point $x \in \mathcal{X}$.

Inequality (1.11) and Proposition 1.13 imply

Proposition 1.16. If a measure $\lambda \in \mathcal{P}(\partial \mathcal{X})$ does not have atoms of weight at least $\frac{1}{2}$, then the infimum in Definition 1.15 is finite, the quasi-barycenter set $\mathcal{C}(\lambda, x, r)$ is compact for any $x \in \mathcal{X}$ and $r>0$, and

$$
\mathcal{C}(\lambda, x, r) \subset \mathcal{C}\left(\lambda, x^{\prime}, r+6 C_{2}\right) \quad \forall x, x^{\prime} \in \mathcal{X} .
$$

DeFinition 1.17. We shall call the closure

$$
\mathcal{C}(\lambda, r)=\overline{\bigcup_{x \in \mathcal{X}} \mathcal{C}(\lambda, x, r)}
$$

the quasi-barycenter set of the measure $\lambda \in \mathcal{P}(\partial \mathcal{X})$.

Proposition 1.16 then implies (cf. Ada96 Proposition 5.1]):

Proposition 1.18. If a measure $\lambda \in \mathcal{P}(\partial \mathcal{X})$ does not have atoms of weight at least $\frac{1}{2}$, then for any $r>0$ the quasi-barycenter set $\mathcal{C}(\lambda, r)$ is compact, and the map $\lambda \mapsto \mathcal{C}(\lambda, r)$ is $\operatorname{Iso}(\mathcal{X})$-equivariant.

1.3. Bounded geometry conditions. Below we shall use several bounded geometry type conditions on a metric space $\mathcal{X}$.

Definition 1.19. We say that a Radon measure $m$ on a metric space $\mathcal{X}$ is $\left(r_{1}, r_{2}\right)$-tempered (with $0<r_{1} \leq r_{2} \leq \infty$ ) if for any $r \in\left[r_{1}, r_{2}\right]$ the measures of closed $r$-balls in $\mathcal{X}$ are uniformly bounded from above and bounded away from 0 , i.e., there exist constants $0<\underline{C}(r) \leq \bar{C}(r)<\infty, r \in\left[r_{1}, r_{2}\right]$ such that

$$
\underline{C}(r) \leq m B(x, r) \leq \bar{C}(r) \quad \forall x \in \mathcal{X} .
$$

A measure is tempered if it is $(r, \infty)$-tempered for a certain $r$. A metric space $\mathcal{X}$ is tempered if it carries an $\operatorname{Iso}(\mathcal{X})$-invariant tempered measure.

Definition 1.20 (cf. Ada96). A metric space $\mathcal{X}$ is exponentially $\rho$-bounded (with $\rho>0$ ) if there exists a constant $a=a(\rho)$ such that, for every $x \in \mathcal{X}$ and every $r>0$, the ball $B(x, r)$ in $\mathcal{X}$ can contain at most $a^{r}$ pairwise disjoint balls of radius $\rho$. We shall say that $\mathcal{X}$ is exponentially bounded if it is exponentially $\rho$-bounded for a certain $\rho$. 
REMARK 1.21. Our terminology differs from that of [Ada96. What we call "exponentially bounded" is "at most exponential" in Ada96.

Theorem 1.22. A proper geodesic metric space $\mathcal{X}$ is exponentially bounded if and only if it is tempered.

The proof consists in checking two following easy claims.

Proposition 1.23. If a geodesic metric space $\mathcal{X}$ carries a $\left(r_{1}, r_{2}\right)$-tempered measure with $r_{2} / r_{1}>3$, then it is exponentially $r_{1}$-bounded.

Proof. For simplicity put $\rho=r_{1}$ and $\varepsilon=r_{2}-3 r_{1}$. For any $r \geq \rho$ let $N_{r}$ be the supremum (over $x \in \mathcal{X}$ ) of cardinalities of disjoint systems of $\rho$-balls in the balls $B(x, r)$. We shall show that the growth of $N_{r}$ is at most exponential.

Let $\left\{x_{i}\right\}_{i=1}^{N}$ be a maximal system of points in the ball $B(x, r)$ such that the balls $B\left(x_{i}, \rho\right)$ are pairwise disjoint. Since this system is maximal, for any point $x^{\prime} \in B(x, r-\rho)$ there is a point $x_{i}$ with $d\left(x^{\prime}, x_{i}\right) \leq 2 \rho$, which means that $2 \rho$-balls centered at the points $x_{i}$ cover the ball $B(x, r-\rho)$, and, therefore, $r_{2}$-balls centered at $x_{i}$ cover the ball $B(x, r+\varepsilon)$ for any $\varepsilon>0$. Thus,

$$
m B(x, r+\varepsilon) \leq N \bar{C}\left(r_{2}\right) \leq N_{r} \bar{C}\left(r_{2}\right) .
$$

On the other hand, the cardinality of any disjoint system of $\rho$-balls in $B(x, r+\varepsilon)$ obviously does not exceed the ratio $m B(x, r+\varepsilon) / \underline{C}(\rho)$, whence

$$
\frac{N_{r+\varepsilon}}{N_{r}} \leq \frac{\bar{C}\left(r_{2}\right)}{\underline{C}\left(r_{1}\right)}
$$

which implies the claim.

Proposition 1.24 (cf. Ada96 Section 6.2]). If a geodesic metric space $\mathcal{X}$ is exponentially $\rho$-bounded, then it carries a $(9 \rho, \infty)$-tempered measure. Moreover, if $\mathcal{X}$ is proper, then such a measure can be chosen to be $\operatorname{Iso}(\mathcal{X})$-invariant.

Proof. Without the requirement of $\operatorname{Iso}(\mathcal{X})$-invariance the measure in question can be easily taken to be, for instance, the sum of $\delta$-measures on a maximal $3 \rho$ separated system of points in $\mathcal{X}$. However, in order to obtain an $\operatorname{Iso}(\mathcal{X})$-invariant measure a little bit more work has to be done.

Since the space $\mathcal{X}$ is proper, the group $\operatorname{Iso}(\mathcal{X})$ is locally compact with respect to the pointwise convergence topology. For any orbit $\mathcal{O}=\operatorname{Iso}(\mathcal{X}) o, o \in \mathcal{X}$, choose the left-invariant Haar measure $\mathfrak{m}_{\mathcal{O}}$ on $\operatorname{Iso}(\mathcal{X})$ normalized by the condition

$$
\mathfrak{m}_{\mathcal{O}}\{g: d(o, g o) \leq 6 \rho\}=1,
$$

and denote by $m_{\mathcal{O}}$ the image of $\mathfrak{m}_{\mathcal{O}}$ under the map $g \mapsto g o$. Obviously, the measure $m_{\mathcal{O}}$ is $\operatorname{Iso}(\mathcal{X})$-invariant, it does not depend on the choice of the point $o$ from the orbit $\mathcal{O}$, and

$$
m_{\mathcal{O}} B(x, 6 \rho)=1 \quad \forall x \in \mathcal{O} .
$$

Now take a maximal system of $\operatorname{Iso}(\mathcal{X})$-orbits $\mathcal{O}_{i}$ in $\mathcal{X}$ with the property that the pairwise distances between different orbits from this system are at least $3 \rho$. The measure $m=\sum_{i} m_{\mathcal{O}_{i}}$ is clearly $\operatorname{Iso}(\mathcal{X})$-invariant. We claim that it is $(9 \rho, \infty)$ tempered. 
First of all, since the system $\left\{\mathcal{O}_{i}\right\}$ is maximal, any point $x \in \mathcal{X}$ lies within the distance $3 \rho$ from at least one of the orbits $\mathcal{O}_{i}$. Therefore, the ball $B(x, 9 \rho)$ contains a $6 \rho$-ball centered at a point $x^{\prime} \in \mathcal{O}_{i}$, so that

$$
m B(x, 9 \rho) \geq m_{\mathcal{O}_{i}} B\left(x^{\prime}, 6 \rho\right) \geq 1
$$

by the definition of the measures $m_{\mathcal{O}_{i}}$.

In order to obtain an upper estimate for the measures $m B(x, r)$ first notice that the exponential $\rho$-boundedness of the space $\mathcal{X}$ implies that the ball $B(x, r)$ can be covered by at most $e^{a r}$ balls $B\left(x_{i}, 3 \rho\right)$ (see the beginning of the proof of Proposition 1.3). Thus, for any $\operatorname{Iso}(\mathcal{X})$-orbit $\mathcal{O}$ the intersection $B(x, r) \cap \mathcal{O}$ can be covered by at most $e^{a r}$ balls of radius $6 \rho$ centered at points from $\mathcal{O}$. Therefore,

$$
m_{\mathcal{O}} B(x, r) \leq e^{a r} .
$$

It remains to notice that the ball $B(x, r)$ can only intersect a uniformly bounded number of orbits $\mathcal{O}_{i}$. Indeed, choose a point $x_{i}$ on each orbit $\mathcal{O}_{i}$ intersecting $B(x, r)$. Then the pairwise distances between the points $x_{i}$ are at least $3 \rho$ by the definition of the system $\left\{\mathcal{O}_{i}\right\}$. Therefore, the $\rho$-balls centered at the points $x_{i}$ are all pairwise disjoint and contained in $B(x, r+\rho)$, so that by the exponential $\rho$-boundedness of the space $\mathcal{X}$ the number of these balls (and therefore of the orbits $\mathcal{O}_{i}$ intersecting $B(x, r))$ does not exceed $e^{a(r+\rho)}$. Combining this estimate with the inequality (1.26) gives

$$
m B(x, r) \leq e^{a(2 r+\rho)} .
$$

Another bounded geometry type condition which can be imposed on a group of isometries of a metric space is that of finiteness of the critical exponent.

Definition 1.27 (BM96). Given a positive Radon measure $m$ on a metric space $\mathcal{X}$, the number (possibly infinite!)

$$
\delta_{c r}(m)=\inf \left\{\delta \geq 0: \int_{\mathcal{X}} e^{-\delta d(o, x)} d m(x)<\infty\right\}
$$

is independent of the choice of the reference point $o \in \mathcal{X}$, and is called the critical exponent of the measure $m$. The critical exponent $\delta_{c r}(G)$ of a closed subgroup $G \subset \operatorname{Iso}(\mathcal{X})$ is by definition the critical exponent of a positive $G$-invariant measure supported on a $G$-orbit in $\mathcal{X}$. Equivalently, for any two points $o, x \in \mathcal{X}$

$$
\delta_{c r}(G)=\inf \left\{\delta \geq 0: \int_{G} e^{-\delta d(o, g x)} d \mathfrak{m}(g)<\infty\right\},
$$

where $\mathfrak{m}$ is a left-invariant Haar measure on $G$.

Generally speaking, finiteness of the critical exponent of the group $\operatorname{Iso}(\mathcal{X})$ does not imply exponential boundedness of the space $\mathcal{X}$, because the orbits of $\operatorname{Iso}(\mathcal{X})$ may be "too small" to provide any information about the whole space $\mathcal{X}$. However, the converse implication is easily seen to be true:

Proposition 1.29. If the space $\mathcal{X}$ is exponentially bounded, then the critical exponent of any closed subgroup $G \subset \operatorname{Iso}(\mathcal{X})$ is finite. 
Proof. Fix a $G$-invariant measure $m$ on the $G$-orbit of a reference point $o \in \mathcal{X}$. By changing variables the integral (1.28) from the definition of the critical exponent can be presented as

$$
\begin{aligned}
\int_{\mathcal{X}} e^{-\delta d(o, x)} d m(x) & =\int_{0}^{1} m\left\{x: e^{-\delta d(o, x)} \geq t\right\} d t \\
& =\delta \int_{0}^{\infty} m B(o, r) e^{-\delta r} d r .
\end{aligned}
$$

Since exponential boundedness of $\mathcal{X}$ implies that $m B(o, r)$ is dominated by an exponential function of $r$ (cf. the proof of Proposition 1.24), the integral (1.30) is finite if $\delta$ is big enough.

REMARK 1.31. In fact, it would be sufficient to look just at the full group Iso $(\mathcal{X})$, because for any closed subgroup its critical exponent does not exceed that of the group Iso $(\mathcal{X})$, see [BM96 Lemma 1.5].

Remark 1.32. If the group $\operatorname{Iso}(\mathcal{X})$ acts on $\mathcal{X}$ cocompactly, then $\mathcal{X}$ is easily seen to be exponentially bounded (because the exponential rate of growth of the locally compact group Iso $(\mathcal{X})$ is finite), which by Proposition 1.29 implies finiteness of the critical exponent of Iso( $\mathcal{X})$ (cf. [BM96, Proposition 1.7]).

\subsection{Boundary maps.}

TheOREm 1.33. For any exponentially bounded hyperbolic space $\mathcal{X}$ there exists a sequence of $\operatorname{Iso}(\mathcal{X})$-equivariant Borel maps $\lambda_{n}: \mathcal{X} \times \partial \mathcal{X} \rightarrow \mathcal{P}(\mathcal{X})$ such that

$$
\left\|\lambda_{n}(x, \gamma)-\lambda_{n}\left(x^{\prime}, \gamma\right)\right\| \rightarrow 0 \quad \forall x, x^{\prime} \in \mathcal{X}, \gamma \in \partial \mathcal{X} .
$$

For proving Theorem 1.33 we shall need the following simple inequality:

Lemma 1.35. Let $\left\{Z_{k}\right\}_{k=1}^{\infty},\left\{Z_{k}^{\prime}\right\}_{k=1}^{\infty}$ be two increasing sequences of measurable sets of finite positive measure in a measure space $(\mathcal{X}, m)$ such that for a certain integer $\tau>0$

$$
Z_{k} \subset Z_{k+\tau}^{\prime}, \quad Z_{k}^{\prime} \subset Z_{k+\tau} \quad \forall k \geq 1,
$$

and let

$$
\lambda_{n}=\frac{1}{n} \sum_{k=1}^{n} m_{Z_{k}}, \quad \lambda_{n}^{\prime}=\frac{1}{n} \sum_{k=1}^{n} m_{Z_{k}^{\prime}}
$$

be the Cesaro averages of the sequences $\left\{m_{Z_{k}}\right\},\left\{m_{Z_{k}^{\prime}}\right\}$, respectively. Then

$$
\left\|\lambda_{n}-\lambda_{n}^{\prime}\right\| \leq \frac{2 \tau}{n}+\frac{4(n-\tau)}{n}\left[1-\left(\frac{m Z_{1}}{m Z_{n+\tau}}\right)^{\frac{2 \tau}{n-\tau}}\right] \quad \forall n>\tau .
$$

Proof. First notice that for any $k>\tau$

$$
\left\|m_{Z_{k-\tau}}-m_{Z_{k}^{\prime}}\right\|=2\left(1-\frac{m Z_{k-\tau}}{m Z_{k}^{\prime}}\right) \leq 2\left(1-\frac{m Z_{k-\tau}}{m Z_{k+\tau}}\right),
$$

and the same inequality holds for $\left\|m_{Z_{k-\tau}}-m_{Z_{k}}\right\|$, so that

$$
\left\|m_{Z_{k}}-m_{Z_{k}^{\prime}}\right\| \leq 4\left(1-\frac{m Z_{k-\tau}}{m Z_{k+\tau}}\right) \quad \forall k>\tau .
$$


Then

$$
\begin{aligned}
\left\|\lambda_{n}-\lambda_{n}^{\prime}\right\| \leq \frac{1}{n} \sum_{k=1}^{n}\left\|m_{Z_{k}}-m_{Z_{k}^{\prime}}\right\| & \leq \frac{2 \tau}{n}+\frac{4}{n} \sum_{k=\tau+1}^{n}\left(1-\frac{m Z_{k-\tau}}{m Z_{k+\tau}}\right) \\
& \leq \frac{2 \tau}{n}+\frac{4(n-\tau)}{n}\left[1-\left(\prod_{k=\tau+1}^{n} \frac{m Z_{k-\tau}}{m Z_{k+\tau}}\right)^{\frac{1}{n-\tau}}\right]
\end{aligned}
$$

whence the claim.

CoROLlary 1.37. If in Lemma 1.35 the growth of the sets $Z_{n}$ with respect to the measure $m$ is subexponential, i.e., $\left(m Z_{n}\right)^{1 / n} \rightarrow 1$, then $\left\|\lambda_{n}-\lambda_{n}^{\prime}\right\| \rightarrow 0$.

Proof of Theorem 1.33, Given points $x \in \mathcal{X}, \gamma \in \partial \mathcal{X}$ and positive integers $n \geq k \geq 1$ put

$$
Y(x, \gamma, n, k)=\{\xi(n): \xi \in \mathcal{R}, d(x, \xi(0)) \leq k, \xi(\infty)=\gamma\},
$$

where $\mathcal{R}$ is the set of all geodesic rays in $\mathcal{X}$. We shall fix a $(r, \infty)$-tempered $\operatorname{Iso}(\mathcal{X})$ invariant measure $m$ on $\mathcal{X}$ provided by Theorem 1.22 and denote by $Z(x, \gamma, n, k)$ the closed $r$-neighbourhood of $Y(x, \gamma, n, k)$.

As it follows from Proposition [1.3 for any ray $\eta$ joining $x$ and $\gamma$ the set $Z(x, \gamma, n, k)$ is contained in the closed $\left(C_{1}+r\right)$-neighbourhood of the geodesic segment $\eta([n-k, n+k])$. Therefore, since the measure $m$ is $(r, \infty)$-tempered, the values $m Z(x, \gamma, n, k)$ are bounded away from 0 and uniformly bounded from above by an affine function of $k$.

For any $\tau \geq d\left(x, x^{\prime}\right)$ the finite sequences of sets

$$
\{Z(x, \gamma, n, k)\}_{k=1}^{n} \quad \text { and } \quad\left\{Z\left(x^{\prime}, \gamma, n, k\right)\right\}_{k=1}^{n}
$$

are $\tau$-sandwiched in the sense of formula (1.36), whence, by putting

$$
\lambda_{n}(x, \gamma)=\frac{1}{n} \sum_{k=1}^{n} m_{Z(x, \gamma, n, k)}
$$

and applying Lemma 1.35 we obtain the claim.

THEOREM 1.38. If $G$ is a closed group of isometries of a CAT $(-1)$ space $\mathcal{X}$ with finite critical exponent, then there exists a sequence of $G$-equivariant Borel maps $\lambda_{n}: \mathcal{X} \times \partial \mathcal{X} \rightarrow \mathcal{P}(\mathcal{X})$ such that

$$
\left\|\lambda_{n}(x, \gamma)-\lambda_{n}\left(x^{\prime}, \gamma\right)\right\| \rightarrow 0 \quad \forall x, x^{\prime} \in \mathcal{X}, \gamma \in \partial \mathcal{X} .
$$

Proof. Let us fix a number $\delta>\delta_{c r}$, a reference point $o \in \mathcal{X}$ and a $G$-invariant Radon measure $m$ on the orbit Go. For any $x \in \mathcal{X}$ denote by $\nu_{x}=\nu_{x}^{\delta}$ the probability measure on the orbit Go with the density

$$
\frac{e^{-\delta d(x, y)}}{\int_{\mathcal{X}} e^{-\delta d(x, y)} d m(y)}
$$

with respect to the measure $m$. We shall call the measures $\nu_{x}$ pre-Patterson as their limits when $\delta$ tends to the critical exponent $\delta_{c r}$ provide the famous Patterson measures on the boundary $\partial \mathcal{X}$, see Pat76, BM96. Obviously, the map $x \mapsto \nu_{x}$ is $G$-equivariant. This fact, together with the local Lipschitz continuity of the map $x \mapsto \nu_{x}$ and boundary convergence of geodesics (guaranteed by the CAT $(-1)$ condition) yields the proof. 
Since for any $x, x^{\prime}, y \in \mathcal{X}$

$$
e^{-\delta d\left(x, x^{\prime}\right)} \leq \frac{e^{-\delta d\left(x^{\prime}, y\right)}}{e^{-\delta d(x, y)}} \leq e^{\delta d\left(x, x^{\prime}\right)},
$$

the definition of the pre-Patterson measures implies that

$$
\left\|\nu_{x}-\nu_{x^{\prime}}\right\| \leq 3 \delta d\left(x, x^{\prime}\right)
$$

provided $d\left(x, x^{\prime}\right)$ is small enough.

Now put

$$
\lambda_{n}(x, \gamma)=\frac{1}{n} \int_{0}^{n} \nu_{\xi(t)} d t
$$

where $\xi$ is the geodesic ray joining $x$ and $\gamma$. By the $\mathrm{CAT}(-1)$ property for any other point $x^{\prime} \in \mathcal{X}$

$$
d\left(\xi(t), \xi^{\prime}(t+T)\right) \underset{t \rightarrow \infty}{\longrightarrow} 0
$$

where $\xi^{\prime}$ is the geodesic ray joining $x^{\prime}$ and $\gamma$, and $T$ is the value of the Busemann cocycle $\beta_{\gamma}\left(x, x^{\prime}\right)$ (see Remark 1.14), which in combination with formula (1.40) implies the claim.

REMARK 1.41. It can be easily seen from the proofs of Theorem 1.33 and Theorem 1.38 that for any compact subset $K \subset \mathcal{X}$ the convergence in formulas (1.34) and (1.39), respectively, is uniform on $K \times K \times \partial \mathcal{X}$.

\section{Hyperbolic equivalence relations}

2.1. Graphed equivalence relations. We begin with recalling the basic notions of the theory of discrete equivalence relations, see FM77. Let $X$ be a standard Borel space. An equivalence relation $R$ on $X$ is called standard if it is a Borel subset of $X \times X$, and it is called countable if the equivalence class (the leaf) $[x]=R(x)=\{y:(x, y) \in R\}$ of any point $x \in X$ is at most countable. A standard countable equivalence relation is also called discrete.

Proposition 2.1 (cf. [FM77 proof of Theorem 1]). For any discrete equivalence relation $R$ with infinite equivalence classes there exists a sequence $\left\{\alpha_{n}\right\}_{n=1}^{\infty}$ of measurable maps $\alpha_{n}: X \rightarrow X$ such that their graphs are pairwise disjoint and their union is $R$. In other words, for any $x \in X$ the sequence $\alpha_{n}(x)$ determines an ordering of the class $[x]$, and these orderings depend on $x$ measurably.

A standard equivalence relation $R$ is called non-singular with respect to a Borel probability measure $\mu$ on $X$ (or, equivalently, the measure $\mu$ is quasi-invariant with respect to $R$ ) if for any subset $A \subset X$ with $\mu(A)=0$ its saturation $[A]=\bigcup_{x \in A}[x]$ also has measure 0 . A non-singular equivalence relation $(X, \mu, R)$ is ergodic if there are no non-trivial saturated sets, it is conservative if all ergodic components are uncountable, and it is dissipative (or of type I) if any ergodic component consists of a single equivalence class, or, equivalently, there exists a leafwise constant measurable $\operatorname{map} \varphi: X \rightarrow X$ with $(x, \varphi(x)) \in R$ for a.e. $x \in X$. In particular, dissipative ergodic equivalence relations are precisely those which consist of a single class. Any nonsingular equivalence relation can be uniquely decomposed into a disjoint union of its conservative and dissipative parts.

Integrating the counting measures on the fibres of the left $(x, y) \mapsto x$ and the right $(x, y) \mapsto y$ projections from $R$ onto $X$ by the measure $\mu$ gives the left $d \mathrm{M}(x, y)=d \mu(x)$ and the right $d \check{\mathrm{M}}(x, y)=d \mathrm{M}(y, x)=d \mu(y)$ counting measures 
on $R$, respectively. The measures $\mathrm{M}$ and $\check{\mathrm{M}}$ are equivalent iff $\mu$ is quasi-invariant. If $\mathrm{M}=\check{\mathrm{M}}$, then the measure $\mu$ is called $R$-invariant.

A (non-oriented) graph structure on an equivalence relation $(X, \mu, R)$ is determined by a measurable subset $K \subset R$ which is symmetric and does not intersect the diagonal $\{(x, x)\} \subset R$. Two points $x, y \in X$ are then joined with an edge iff $(x, y) \in K$. We shall call $(X, \mu, R, K)$ a graphed equivalence relation Ada90. Denote by $[x]_{K}$ the equivalence class $[x]$ endowed with the graph structure $K$.

Definition 2.2. A graphed equivalence relation $(X, \mu, R, K)$ is hyperbolic if a.e. leafwise graph $[x]_{K}$ is a hyperbolic graph (i.e., is Gromov hyperbolic with respect to the graph metric assigning length 1 to all edges). By $\overline{[x]}_{K}$ and $\partial[x]_{K}$ we denote the hyperbolic compactification of the graph $[x]_{K}$ and its boundary, respectively. As usual, $C\left(\overline{[x]}_{K}\right)$ denotes the space of continuous real valued functions on $\overline{[x]}_{K}$. Below we shall always deal with a fixed graph structure $K$ and usually omit the corresponding subscript.

REMARK 2.3. The function assigning to any point $x \in X$ the hyperbolicity constant of the graph $[x]$ is easily seen to be measurable (because the hyperbolicity constant is defined in terms of geodesic triangles in $[x]$ ). Being leafwise constant, this function must therefore be constant on the ergodic components of $R$.

Examples of hyperbolic equivalence relations include, in particular,

(1) the leafwise Cayley graphs of a free action of a word hyperbolic group, see Example 2.16 for more details;

(2) equivalence relations on transversals to hyperbolic foliations, see below Section 2.6

(3) treed equivalence relations (for which a.e. leafwise graph is a tree), see Ada90;

(4) equivalence relations associated with certain fractal sets, see Kai02.

In all these examples the hyperbolic boundaries of different equivalence classes can be naturally identified with (a subspace of) the same topological space (see the discussion of these examples at the end of Section [2.3). However, there is no reason for this to be the case in general (it would be interesting to have an explicit example of this kind), which is why we shall need a technique allowing one to deal with the general situation.

2.2. The bundle of leafwise continuous functions. Recall that a measurable structure on a bundle of Banach spaces $\left\{E_{x}\right\}$ over a Lebesgue (三 standard measure) space $(X, \mu)$ is a family $\mathfrak{M}$ of sections (called measurable) $\sigma: x \mapsto \sigma_{x} \in E_{x}$ such that

(i) the function $x \mapsto\left\|\sigma_{x}\right\|$ is measurable for any section $\sigma \in \mathfrak{M}$;

(ii) the family $\mathfrak{M}$ is closed under addition, multiplication by measurable scalar functions and passing to pointwise limits in the norm topology.

The couple $\left(\left\{E_{x}\right\}, \mathfrak{M}\right)$ is then called a measurable bundle of Banach spaces over $(X, \mu)$, see [FD88, Chapter II.4], ADR00 Appendix A.3]. A measurable bundle $\left(\left\{E_{x}\right\}, \mathfrak{M}\right)$ is separable if there exists a sequence of sections $\sigma^{n} \in \mathfrak{M}$ such that for a.e. $x \in X$ the sequence $\left\{\sigma_{x}^{n}\right\}$ is dense in $E_{x}$.

Proposition 2.4. Given a hyperbolic equivalence relation $(X, \mu, R, K)$, denote by $\mathfrak{M}=\mathfrak{M}(X, \mu, R, K)$ the space of all measurable functions $F: R \rightarrow \mathbb{R}$ such 
that for a.e. $x \in X$ the function $F(x, \cdot)$ extends to a continuous function on the completion $\overline{[x]}$. Then $\mathfrak{M}$ is a measurable structure on the bundle of Banach spaces $\{C(\overline{[x]})\}, x \in X$, and the measurable bundle $(\{C(\overline{[x]})\}, \mathfrak{M})$ is separable.

Proof. Verification of the conditions from the definition of a measurable structure being straightforward, we only have to check the separability. Since the leafwise hyperbolic compactifications $\overline{[x]}$ are completions of the equivalence classes $[x]$ with respect to the metrics $\rho_{x}$ (1.4), and these metrics depend on $x$ measurably, the functions

$$
F_{n}(x, y)=\rho_{x}\left(y, \alpha_{n}(x)\right)
$$

where $\alpha_{n}$ are the maps from Proposition 2.1 belong to $\mathfrak{M}$. For any $x \in X$ the family of functions $\left\{F_{n}(x, \cdot)\right\}$ separates points of $\overline{[x]}$. By adding to this family the constant function and applying the Weierstraß-Stone theorem we obtain the claim.

The dual bundle $\left(\left\{E_{x}^{*}\right\}, \mathfrak{M}^{*}\right)$ of a separable measurable bundle $\left(\left\{E_{x}\right\}, \mathfrak{M}\right)$ is the bundle of dual Banach spaces $E_{x}^{*}$ endowed with the weak $k^{*}$ measurable structure $\mathfrak{M}^{*}$ which consists of all sections $\sigma^{*}: x \mapsto \sigma_{x}^{*} \in E_{x}^{*}$ such that the map $x \mapsto\left\langle\sigma_{x}, \sigma_{x}^{*}\right\rangle$ is measurable for any section $\sigma \in \mathfrak{M}$, see [ADR00 Lemma A.3.7].

Returning to the measurable bundle $(\{C(\overline{[x]})\}, \mathfrak{M})$ of leafwise continuous functions, which is associated with the hyperbolic equivalence relation $(X, \mu, R, K)$, notice that for any space $C(\overline{[x]})$ its dual is the space $M(\overline{[x]})$ of signed Borel measures (equipped with the total variation norm) on the completion $\overline{[x]}$. Therefore, in view of Proposition 2.4 we have a measurable structure $\mathfrak{M}^{*}$ on the bundle $\{M(\overline{[x]})\}$. Denote by $\mathfrak{M}_{\circ}^{*}$ and $\mathfrak{M}_{\partial}^{*}$ the measurable structures induced by $\mathfrak{M}^{*}$ on the interior subbundle $\{M([x])\}$ and the boundary subbundle $\{M(\partial[x])\}$, respectively.

REMARK 2.5. One can easily check that the splitting of the bundle $\{M(\overline{[x]})\}$ into the direct sum of the interior and boundary subbundles is measurable in the sense that for any measurable section of $\{M(\overline{[x]})\}$ its interior and boundary parts are measurable too. Also, the Hahn decomposition of any measurable section of $\{M(\overline{[x]})\}$ into the difference of its positive and negative parts is measurable.

Definition 2.6. Measurable sections of the bundle $\left(\{M([x])\}, \mathfrak{M}_{\circ}^{*}\right)$ (resp., of the bundle $\left(\{M(\partial[x])\}, \mathfrak{M}_{\partial}^{*}\right)$ ) are called measurable systems of interior (resp., boundary) measures on a hyperbolic equivalence relation $(X, \mu, R, K)$.

2.3. Measurable systems of boundary measures. Since the functions

$$
\Delta_{n}(x, y)= \begin{cases}1, & y=\alpha_{n}(x), \\ 0, & \text { otherwise }\end{cases}
$$

where $\alpha_{n}$ are the maps from Proposition 2.1 obviously belong to $\mathfrak{M}$, the measurable structure $\mathfrak{M}_{\circ}^{*}$ of the interior bundle $\{M([x])\}$ consists precisely of those systems of measures $\left\{\lambda_{x}\right\}$ for which $\lambda_{x}(y)$ is measurable as a function on $R$. We shall now give an explicit description of the measurable structure $\mathfrak{M}_{\partial}^{*}$ of the boundary bundle $\{M(\partial[x])\}$.

TheOREM 2.7. The measurable structure $\mathfrak{M}_{\partial}^{*}$ of the boundary bundle consists of pointwise weak* limits (in the spaces $M(\overline{[x]}), x \in X$ ) of sequences of measurable sections of the interior bundle $\{M([x])\}$. 
Proof. The definition of the dual bundle implies that its measurable structure $\mathfrak{M}^{*}$ is closed with respect to passing to pointwise weak* limits, so that we only have to prove that any measurable system of boundary measures $\lambda \in \mathfrak{M}_{\partial}^{*}$ can be approximated by a sequence of measurable systems of interior measures $\lambda^{n} \in \mathfrak{M}_{\circ}^{*}$. In other words, for any boundary measure $\lambda_{x} \in M(\partial[x])$ we have to construct a sequence of interior measures $\lambda_{x}^{n} \in M([x])$ converging to $\lambda_{x}$, and to do it in a measurable way with respect to $x$.

Let us begin with the following observation. Any finite continuous partition of unity

$$
\mathbf{1}=\sum_{i \in I} \varphi_{i}
$$

on a compact space $K$ determines a norm 1 linear map $\pi$ from the space $M(K)$ of (signed) Borel measures on $K$ to the space $M(I)$ of (signed) measures on the index set $I$ by the formula

$$
\pi \theta(i)=\left\langle\varphi_{i}, \theta\right\rangle
$$

Suppose now that for any $x \in X$ and any integer $n$ we have a partition of unity on the completion $\overline{[x]}$ parameterized by the points from the $n$-sphere of the leafwise graph metric $S(x, n) \subset[x]$. These partitions then determine a sequence of maps $\pi_{n}$ from $M(\overline{[x]})$ to $M(S(x, n))$. We shall show that these partitions of unity can be constructed measurably with respect to $x$, and in such a way that $\pi_{n} \theta \rightarrow \theta$ for any boundary measure $\theta \in M(\partial[x])$.

For the moment we fix a point $x \in X$, an integer $n>0$, and a real $\varepsilon>0$. Recall that the hyperbolic compactification of the equivalence class $[x]$ is its completion with respect to the metric $\rho_{x}$ (1.4). Now for any $s \in S=S(x, n)$ define the non-negative function $f_{s} \in C(\overline{[x]})$ by putting for $z \in \overline{[x]}$

$$
f_{s}(z)=\left\{\begin{array}{lll}
1-\frac{\rho_{x}(z, s)-\rho_{x}(z, S)}{\varepsilon} & , & \rho_{x}(z, s) \leq \rho_{x}(z, S)+\varepsilon ; \\
0 & , \text { otherwise }
\end{array}\right.
$$

Then the functions

$$
\varphi_{s}=\frac{f_{s}}{\sum_{s \in S} f_{s}}
$$

form a partition of unity on $\overline{[x]}$ parameterized by the points $s \in S$, and these functions have the property that

$$
\varphi_{s}(z)=0 \quad \forall z \in \overline{[x]}: \rho_{x}(z, s) \geq \rho_{x}(z, S)+\varepsilon .
$$

As it follows from the definition of the metric $\rho_{x}$ (1.4),

$$
\rho_{x}(\gamma, S)=e^{-a n} \quad \forall \gamma \in \partial[x],
$$

where $a=1 / 15 \delta$. Indeed, $\rho_{x}(y, s) \geq e^{-a n}$ for any two points $y \in[x]$ and $s \in S$, so that $\rho_{x}(\gamma, s) \geq e^{-a n}$. On the other hand, if $\xi$ is a geodesic ray joining $x$ and $\gamma$, then

$$
\rho_{x}(\gamma, \xi(n))=\lim _{m \rightarrow \infty} \rho_{x}(\xi(m), \xi(n))=e^{-a n} .
$$

From now on we shall assume, for the sake of concreteness, that in the above construction

$$
\varepsilon=\varepsilon(n)=e^{-a n} .
$$


Then formulas (2.10) and (2.11) imply that

$$
\varphi_{s}(\gamma)=0 \quad \forall \gamma \in \partial[x]: \rho_{x}(\gamma, s) \geq 2 e^{-a n} .
$$

Denote now by

$$
\pi_{n}: M(\overline{[x]}) \rightarrow M(S)
$$

the map (2.8) from the space of measures on $\overline{[x]}$ to the space of measures on $S=$ $S(x, n)$ determined by the partition of unity $\left\{\varphi_{s}\right\}_{s \in S}$ (2.9). Then by (2.12)

$$
\operatorname{supp} \pi_{n} \delta_{\gamma} \subset S \cap B_{x}\left(\gamma, 2 e^{-a n}\right) \quad \forall \gamma \in \partial[x],
$$

and the linearity of the maps $\pi_{n}$ implies the weak ${ }^{*}$ convergence of the sequence $\pi_{n} \theta$ to $\theta$ for any boundary measure $\theta \in M(\partial[x])$. Obviously, the whole construction is measurable in $x$.

Proposition 2.14. For any measurable system of boundary measures on a hyperbolic equivalence relation its continuous and atomic parts are also measurable.

Proof. In view of Remark 2.5] it is enough to establish the claim for systems of non-negative measures only. For any such system $\lambda=\left\{\lambda_{x}\right\}$ it is sufficient to show that the system $\hat{\lambda}=\left\{\widehat{\lambda}_{x}\right\}$ which consists of maximal weight atoms of the measures $\lambda_{x}$ is also measurable.

We shall use the approximation of the measures $\lambda_{x}$ by the interior measures $\pi_{n} \lambda_{x}$ constructed in the proof of Theorem 2.7 Let us first notice that, as it follows from formulas (2.11) and 2.13), for any point $\gamma \in \partial[x]$ there exists $s \in S(x, n)$ such that

$$
\operatorname{supp} \pi_{n} \delta_{\gamma} \subset S(x, n) \cap B_{x}\left(s, 3 e^{-a n}\right) .
$$

Formulas (2.13) and (2.15) show that the maximal weight of the measure $\lambda_{x}$ can be expressed in terms of the measures $\pi_{n} \lambda_{x}$ as

$$
W(x)=\lim _{n \rightarrow \infty} \max _{s \in S(x, n)}\left(\pi_{n} \lambda_{x}\right)\left[B_{x}\left(s, 3 e^{-a n}\right)\right],
$$

and, in particular, the function $x \mapsto W(x)$ is measurable. Let us now denote by $\widehat{\lambda}_{x}^{n}$ the restriction of the measure $\pi_{n} \lambda_{x}$ to the $3 e^{-a n}$-neighbourhood (in the metric $\rho_{x}$ ) of the set

$$
\left\{s \in S(x, n):\left(\pi_{n} \lambda_{x}\right)\left[B_{x}\left(s, 3 e^{-a n}\right)\right] \geq W(x)\right\} .
$$

Then for any $x \in X$ the sequence $\widehat{\lambda}_{x}^{n}$ converges (in the weak* topology) to the measure $\widehat{\lambda}_{x}$. Since for any $n$ the system of measures $\left\{\hat{\lambda}_{x}^{n}\right\}$ is measurable, by Theorem 2.7 the system $\widehat{\lambda}_{x}$ is also measurable.

In the situations when the hyperbolic boundaries of different equivalence classes can be identified with (a subspace of) the same topological space, the notion of measurability introduced above coincides with the "usual" measurability as one can easily verify by using Theorem 2.7

EXAMPLE 2.16. Recall that the classes of the orbit equivalence relation $R_{G}$ of a measure class preserving action of a countable group $G$ on a measure space $(X, \mu)$ are the orbits of the action. Any symmetric generating set $A \subset G$ determines the graph structure $K=\{(x, g x): x \in X, g \in A\}$ on $R_{G}$. If the action of $G$ is free (mod 0), then the map $g \mapsto g^{-1} x$ establishes an isomorphism of the (right) Cayley graph of $G$ with a.e. graph $[x]$. If the group $G$ is in addition word hyperbolic (i.e., the Cayley graph $(G, A)$ is hyperbolic), then the orbit equivalence relation $R_{G}$ is 
obviously hyperbolic, and the above isomorphism extends to a homeomorphism $\varphi_{x}$ from the hyperbolic boundary $\partial G$ of the group $G$ to the hyperbolic boundary $\partial[x]$ of the equivalence class $[x]$ for a.e. point $x \in X$. Therefore, in this situation $M(\partial[x]) \cong M(\partial G)$ for a.e. $x \in X$, so that the boundary bundle is isomorphic to a constant Banach bundle, and Theorem 2.7 implies that the measurability of a system of boundary measures $\lambda_{x} \in M(\partial[x])$ in the sense of Definition [2.6] is equivalent to the measurability of the map $x \mapsto \varphi_{x}^{-1}\left(\lambda_{x}\right)$ from $X$ to $M(\partial G)$.

EXAmple 2.17. Any simply connected Riemannian manifold $M$ with pinched negative sectional curvatures is $\delta$-hyperbolic, and the exponential map at any point $x \in M$ establishes a homeomorphism between the unit tangent sphere at the point $x$ and the hyperbolic boundary of $M$ (in this situation the hyperbolic compactification coincides with the visibility compactification). Therefore, given a hyperbolic Riemannian measurable foliation, the leafwise hyperbolic boundaries are homeomorphic to spheres, and the boundary bundle for the equivalence relation on any uniform system of transversals (see Section 2.6) is isomorphic to a constant Banach bundle. The measurability of a system of boundary measures on this equivalence relation is then equivalent to the measurability of the corresponding system of measures on the leafwise unit tangent spheres of the foliation.

EXAMPLE 2.18. Yet another example when the boundary bundle is isomorphic to a constant Banach bundle (more precisely, to a subbundle of a constant bundle) is provided by treed equivalence relations (see Ada90) for which a.e. graph $[x]$ is a tree. In this case any such tree can be embedded into the universal countable tree. Once again, Theorem 2.7 implies that the measurability of a system of boundary measures in the sense of Definition 2.6 is equivalent to the measurability of the corresponding map from $X$ to the space of boundary measures of the universal tree.

\subsection{Invariant systems of boundary measures.}

Definition 2.19. A measurable system $\lambda=\left\{\lambda_{x}\right\}$ of boundary measures on a hyperbolic equivalence relation $(X, \mu, R, K)$ is called invariant if it is a.e. constant, i.e., $\lambda_{x}=\lambda_{y}$ for M-a.e. $(x, y) \in R$.

THEOREM 2.20. If $\lambda=\left\{\lambda_{x}\right\}$ is an invariant system of boundary measures on a conservative hyperbolic equivalence relation $(X, \mu, R, K)$, then a.e. measure $\lambda_{x}$ is supported by at most 2 points.

Proof. As it follows from the proof of Proposition 2.14 the set $X^{\prime}$ of all points $x \in X$ with card supp $\lambda_{x} \geq 3$ is measurable, so that without loss of generality we may assume that $X^{\prime}=X$. Further, again by the proof of Proposition 2.14 we may change the system $\lambda$ so that the weights of atoms of a.e. measure $\lambda_{x}$ be strictly less than $\frac{1}{2}$.

The claim is now a straightforward consequence of Proposition 1.16 The only thing we have to take care of is the measurability of the quasi-barycenter set. In order to check it we shall invoke Theorem 2.7 and approximate (in the leafwise weak* topology) the system $\lambda$ by a sequence of measurable systems of interior measures $\lambda^{n}=\left\{\lambda_{x}^{n}\right\}$. Define the integer valued function $\varphi$ on $R$ as

$$
\varphi(x, y)=\limsup _{n \rightarrow \infty} \sum_{z} \beta_{z}(x, y) \lambda_{x}^{n}(z),
$$


where $\beta_{z}$ is the distance cocycle (1.6). Let $Y(x) \subset[x]$ be the set of points where the function $\varphi(x, \cdot)$ attains its minimal value, and put

$$
Z(x)=\bigcup_{x^{\prime} \in[x]} Y\left(x^{\prime}\right) .
$$

Clearly, the set

$$
\{(x, y): x \in X, y \in Z(x)\} \subset R
$$

is measurable, and by Proposition 1.12 and Proposition 1.16 $Z(x)$ is contained in the finite set $\mathcal{C}\left(\lambda, x, 8 C_{2}\right)$ for a.e. $x \in X$. Thus, we have a measurable map assigning to any point $x \in X$ a finite subset $Z(x)$ of the equivalence class $[x]$, which contradicts the conservativity of $R$.

Definition 2.21. A hyperbolic equivalence relation is elementary if there exists a measurable map assigning to a.e. class $[x]$ a finite subset $A[x] \subset \partial[x]$, i.e., if the system of uniform probability measures on the sets $A[x]$ is an invariant measurable system in the sense of Definition 2.6

In the setup of this definition the map $x \mapsto$ card $A[x]$ is measurable (see the proof of Proposition 2.14). Therefore, if an elementary hyperbolic equivalence relation is ergodic, then almost all sets $A[x]$ must have the same cardinality. If $q \geq 1$ is its value, then we shall call the associated system of boundary measures and the equivalence relation q-elementary. Note that even if a hyperbolic equivalence relation is elementary, a.e. leafwise boundary may well be infinite (see the examples below). Theorem 2.20 implies

THEOREM 2.22. Let $(X, \mu, R, K)$ be an ergodic hyperbolic equivalence relation which has more than one equivalence class. Then it admits an invariant measurable system of boundary probability measures if and only if it belongs to one of the following 3 classes:

(i) $(X, \mu, R, K)$ admits a unique invariant measurable system of boundary measures which is 1-elementary;

(ii) $(X, \mu, R, K)$ admits a unique invariant measurable system of boundary measures which is 2-elementary;

(iii) $(X, \mu, R, K)$ admits two disjoint 1-elementary systems of boundary measures, and any invariant system of boundary measures is their convex combination.

2.5. Examples of elementary hyperbolic equivalence relations. We shall now give examples of hyperbolic equivalence relations from each of the types described in Theorem 2.22 Take a non-elementary word hyperbolic group $G$, i.e., such that its hyperbolic boundary $\partial G$ consists of at least 3 points (which implies that $\partial G$ is uncountable, see Gro87]). For instance, one can take for $G$ a nonabelian free group. Put

$$
\begin{gathered}
\partial^{2} G=\partial G \times \partial G \backslash \operatorname{diag}=\left\{\left(\gamma_{1}, \gamma_{2}\right): \gamma_{i} \in \partial G, \gamma_{1} \neq \gamma_{2}\right\}, \\
\partial^{3} G=\left\{\left(\gamma_{1}, \gamma_{2}, \gamma_{3}\right): \gamma_{i} \in \partial G, \gamma_{i} \neq \gamma_{j}\right\},
\end{gathered}
$$

and, finally, let $\widetilde{\partial^{2} G}$ be the symmetrization of $\partial^{2} G$, i.e., the set of unordered pairs of elements $\gamma_{1} \neq \gamma_{2} \in \partial G$. 
Proposition 2.23. The orbit equivalence relation $R_{G}$ of a free measure class preserving action of a word hyperbolic group $G$ on a measure space $(X, \mu)$ is 1elementary (resp., 2-elementary) if and only if there exists a measurable G-equiva-

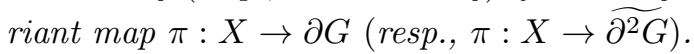

Proof. First notice that the homeomorphisms $\varphi_{x}: \partial G \mapsto \partial[x]$ (see Example 2.16) have the property that $\varphi_{g x}(g \gamma)=\varphi_{x}(\gamma)$ for any $g \in G, \gamma \in \partial G$ and a.e. $x \in X$. Then equivariance of $\pi$ implies that the map $x \mapsto \varphi_{x} \circ \pi(x) \in \partial[x]$ is leafwise constant, i.e., $R_{G}$ is 1-elementary. Conversely, if $R_{G}$ is 1-elementary, and $x \mapsto \Phi(x) \in \partial[x]$ is the corresponding leafwise constant map, then the map $\pi(x)=\varphi_{x}^{-1} \circ \Phi(x)$ is equivariant. With obvious modifications the same argument works in the 2-elementary case as well.

Let us fix a quasi-invariant probability measure $\mu$ on $\partial G$ such that the diagonal action of $G$ on $\left(\partial^{2} G, \mu \otimes \mu\right)$ is ergodic (for instance, the harmonic measure of any non-degenerate symmetric random walk on $G$ has this property, see Kai94). On the other hand, Proposition 1.16 implies that the action of $G$ on $\partial^{3} G$ is proper (cf. Gro87, 8.2.K]). Therefore, this action is dissipative with respect to any quasiinvariant measure.

EXAMPLE 2.24. A hyperbolic equivalence relation with a unique 1-elementary system of boundary measures. This is the orbit equivalence relation of the action of $G$ on $(\partial G, \mu)$.

Proposition 2.23 applied to the identity map on $\partial G$ at once provides us with a 1-elementary system. In order to prove its uniqueness we have, in view of Theorem 2.22 to exclude existence of another 1-elementary system of boundary measures disjoint with the one we have just constructed. By Proposition 2.23 existence of such a system is equivalent to existence of an equivariant map $\pi: \partial G \rightarrow \partial G$ such that a.e. $\pi(\gamma) \neq \gamma$. Such a map $\pi$ would allow one to equivariantly embed $\partial^{2} G$ into $\partial^{3} G$ by the formula $\left(\gamma_{1}, \gamma_{2}\right) \mapsto\left(\gamma_{1}, \pi\left(\gamma_{1}\right), \gamma_{2}\right)$, which is impossible because the action of $G$ on the space $\left(\partial^{2} G, \mu \otimes \mu\right)$ is ergodic, whereas its action on $\partial^{3} G$ (with respect to the image of the measure $\mu \otimes \mu$ ) must be dissipative.

EXAMPLE 2.25. A hyperbolic equivalence relation with a unique system of boundary measures which is 2-elementary. This is the orbit equivalence relation of the action of $G$ on $\widetilde{\partial^{2} G}$ (endowed with the image of the measure $\mu \otimes \mu$ ).

The system in question by Proposition 2.23 corresponds to the identity map on $\widetilde{\partial^{2} G}$. By Theorem 2.22 in order to prove its uniqueness we have to exclude the possibility that this system is the half-sum of two 1-elementary systems, i.e., in view of Proposition [2.23] that there exists an equivariant map $\pi: \widetilde{\partial^{2} G} \rightarrow \partial G$ with $\pi\left(\left\{\gamma_{1}, \gamma_{2}\right\}\right) \subset\left\{\gamma_{1}, \gamma_{2}\right\}$. Such a map $\pi$ would give a $G$-equivariant measurable section of the projection $\partial^{2} G \rightarrow \widetilde{\partial^{2} G}$, which is impossible by the ergodicity of the action of $G$ on $\left(\partial^{2} G, \mu \otimes \mu\right)$.

ExAmple 2.26. A hyperbolic equivalence relation with two 1-elementary systems of boundary measures. This is the orbit equivalence relation of the action of $G$ on $\left(\partial^{2} G, \mu \otimes \mu\right)$.

2.6. Foliations and equivalence relations. Recall that a foliation $\mathcal{F}$ is a smooth manifold decomposed into smooth immersed submanifolds (leaves) organized in a local product structure (we shall use the same notation $\mathcal{F}$ both for the 
foliation and for its underlying manifold). In other words, $\mathcal{F}$ can be covered by flow boxes $U \cong B \times T$ (where $B$ and $T$ are open Euclidean balls) endowed with an additional product structure preserved by the transition maps. The sets $\{x\} \times T, x \in B$ and $B \times\{t\}, t \in T$ are called transversals and plaques, respectively. By $\mathcal{F}(x)$ we denote the leaf of the foliation $\mathcal{F}$ passing through a point $x$. Laminations are defined in precisely the same way except for allowing the transverse structure to be just continuous (rather than smooth), so that for laminations the transversals $T$ are just topological spaces (without any smooth structure). However, the difference between foliations and laminations is insignificant for us, as we shall always (unless otherwise specified) be working in the measure category, and only require measurability of the transverse structure of $\mathcal{F}$ with respect to a certain quasi-invariant transverse measure $\mu$. We shall refer to the couple $(\mathcal{F}, \mu)$ as a measurable foliation (see, for instance, MS88 for more details).

If a measurable foliation $(\mathcal{F}, \mu)$ is endowed with a transversely measurable leafwise Riemannian structure, we shall call it a (leafwise) Riemannian measurable foliation. We shall say that $(\mathcal{F}, \mu)$ is hyperbolic if $\mu$-a.e. leaf is $\delta$-hyperbolic (with respect to its Riemannian metric). A particular case is the situation when a.e. leaf of $\mathcal{F}$ is a simply connected manifold with pinched negative sectional curvatures. In this case measurable systems of boundary measures can be defined as measurable systems of measures on leafwise unit tangent spheres (by identifying the latter with the leafwise hyperbolic $\equiv$ visibility boundaries via the leafwise exponential maps). In the general situation one could apply the approach used for hyperbolic equivalence relations in Section 2.2 and Section 2.3 However, since we are only interested in invariant systems of boundary measures, we shall spare tedious technical details by reducing foliations to equivalence relations on appropriate systems of transversals (such a reduction goes back to Plante Pla75] and Bowen [Bow77]).

Definition 2.27. A system $\mathcal{T}=\left\{T_{i}\right\}$ of transversals of a Riemannian measurable foliation $\mathcal{F}$ is uniform if

(i) For any $r>0$ the number of points from $\mathcal{T}$ in a.e. leafwise ball of radius $r$ is uniformly bounded by a constant $C=C(r)$;

(ii) The intersection of $\mathcal{T}$ with a.e. leaf of $\mathcal{F}$ is non-empty, and there exists a constant $A>0$ such that for any point $x$ of a.e. leaf its leafwise distance to $\mathcal{F}(x) \cap \mathcal{T}$ does not exceed $A$.

A foliation is uniform if it admits a uniform system of transversals.

Compactness considerations immediately imply

Proposition 2.28. Any Riemannian measurable foliation admitting a compact underlying space is uniform.

Let now $(\mathcal{F}, \mu)$ be a uniform Riemannian measurable foliation with a uniform system of transversals $\mathcal{T}$. Denote by $R_{\mathcal{F}}$ the induced equivalence relation on $\mathcal{T}$ (two points are $R_{\mathcal{F}}$-equivalent iff they belong to the same leaf of $\mathcal{F}$ ). Then the restriction $\mu_{\mathcal{T}}$ of the measure $\mu$ to $\mathcal{T}$ is $R_{\mathcal{F}}$-quasi-invariant in the sense of Section 2.1. Consider on $R_{\mathcal{F}}$ the graph structure

$$
K=\left\{(x, y) \in R_{\mathcal{F}}: d(x, y) \leq 3 A\right\},
$$

where $d$ denotes the leafwise Riemannian metric, and $A=A(\mathcal{T})$ is the constant from Definition 2.27 Then one can easily see that the leafwise graph metrics are roughly isometric to the leafwise Riemannian metrics, so that, in particular, 
if $\mathcal{F}$ is hyperbolic, then the graphed equivalence relation $\left(\mathcal{T}, \mu_{\mathcal{T}}, R_{\mathcal{F}}, K\right)$ is also hyperbolic, and the leafwise hyperbolic boundaries with respect to the Riemannian and the graph metrics coincide (e.g., see GdlH90 Proposition 7.14]).

Proposition 2.29. An invariant system of boundary measures on a hyperbolic foliation which is measurable with respect to one uniform system of transversals is also measurable with respect to any other uniform system of transversals.

Proof. Let $\mathcal{T}$ and $\overline{\mathcal{T}}$ be two uniform systems of transversals, and let $\lambda$ be a measurable invariant system of boundary measures of the equivalence relation $\left(\mathcal{T}, R_{\mathcal{F}}\right)$. By Theorem 2.7 we may realize $\lambda$ as the weak* leafwise limit of measurable systems $\lambda^{n}=\left\{\lambda_{x}^{n}\right\}$ of interior measures of $\left(\mathcal{T}, R_{\mathcal{F}}\right)$. We shall need the following "transition maps" allowing one to pass from measures on $\mathcal{T}$ to measures on $\overline{\mathcal{T}}$ and back in a measurable way: denote by $\alpha_{x}, x \in \mathcal{T}$ the probability measure uniformly distributed on the set of points from $\overline{\mathcal{T}}$ nearest to $x$ in the leafwise metric of the leaf $\mathcal{F}(x)$, and by $\bar{\alpha}_{\bar{x}}, \bar{x} \in \overline{\mathcal{T}}$ the probability measure uniformly distributed on the set of points from $\mathcal{T}$ nearest to $\bar{x}$. By the definition of a uniform system of transversals the support of any measure $\alpha_{x}$ lies within a uniformly bounded distance from $x$, and the same property holds for the measures $\bar{\alpha}_{\bar{x}}$ as well. Obviously, both maps $x \mapsto \alpha_{x}$ and $\overline{\boldsymbol{x}} \mapsto \bar{\alpha}_{\bar{x}}$ are measurable. By using the measures $\alpha_{x}$ and $\bar{\alpha}_{\bar{x}}$ one can pass from the sequence $\lambda^{n}$ to the sequence $\bar{\lambda}^{n}$ of measurable systems of interior measures of the equivalence relation $\left(\overline{\mathcal{T}}, R_{\mathcal{F}}\right)$ defined as

$$
\bar{\lambda}_{\bar{x}}^{n}(\bar{y})=\sum_{x, y} \bar{\alpha}_{\bar{x}}(x) \lambda_{x}^{n}(y) \alpha_{y}(\bar{y}) .
$$

Since the measures $\lambda_{x}^{n}$ converge in the weak* topology to a leafwise constant limit, the measures $\bar{\lambda} \frac{n}{x}$ also converge to the same limit, which is therefore measurable with respect to the system of transversals $\overline{\mathcal{T}}$.

By using the above Proposition, we can now define an invariant measurable system of boundary measures of a uniform hyperbolic foliation as an invariant measurable system of boundary measures of any uniform system of transversals. Theorem 2.20] and Theorem 2.22 then imply

THEOREM 2.30. If $\lambda=\left\{\lambda_{x}\right\}$ is an invariant measurable system of boundary measures on a uniform conservative hyperbolic foliation $(\mathcal{F}, \mu)$, then a.e. measure $\lambda_{x}$ is supported by at most 2 points.

THEOREM 2.31. Let $(\mathcal{F}, \mu)$ be a uniform ergodic hyperbolic foliation which has more than one leaf. Then it admits an invariant measurable system of boundary probability measures if and only if it belongs to one of the following 3 classes:

(i) $(\mathcal{F}, \mu)$ admits a unique invariant measurable system of boundary measures which is 1-elementary;

(ii) $(\mathcal{F}, \mu)$ admits a unique invariant measurable system of boundary measures which is 2-elementary;

(iii) $(\mathcal{F}, \mu)$ admits two disjoint 1-elementary invariant measurable systems of boundary measures, and any invariant measurable system of boundary measures is their convex combination.

Examples of foliations from each of the classes described in Theorem 2.31 can be constructed along the same lines as in Section 2.5 


\section{Amenability}

3.1. Groups. The class of amenable groups is a natural generalization of the class of finite groups. We shall remind just two definitions of amenability of a locally compact group $G$ (among a host of other definitions, see Gre69, Pat88, Pie84). The Reiter condition

There exists an approximatively invariant sequence of probability measures $\theta_{n}$ on $G$, i.e., such that $\left\|g \theta_{n}-\theta_{n}\right\| \rightarrow 0$ for any $g \in G$.

by its constructiveness is very useful for verifying amenability of a given group, whereas, on the contrary, the fixed point condition

Any continuous affine action of $G$ on a compact space has a fixed point.

is the one which is most useful for applications of amenability. For instance, the fixed point condition implies at once that any continuous action of an amenable group on a compact space has an invariant measure.

We shall illustrate how these two definitions work together on the example of the following result. Recall that a group of isometries of a hyperbolic space $\mathcal{X}$ is called elementary if it either is compact or fixes a finite subset of $\partial \mathcal{X}$ (actually, if in the latter case the fixed set contains more than 2 points, then the group fixes also a compact subset of $\mathcal{X}$, and therefore is compact, see Proposition 1.18.

TheOREM 3.3. A closed subgroup $G$ of the group of isometries of an exponentially bounded hyperbolic space $\mathcal{X}$ is amenable if and only if it is elementary.

Proof. If $G$ is amenable, then by the fixed point property (3.2) it has an invariant probability measure $\lambda$ on $\partial \mathcal{X}$. If $\lambda$ does not have atoms of weight at least

$\frac{1}{2}$, then by Proposition 1.18 its quasi-barycenter set is compact and fixed by $G$ together with the measure $\lambda$. Otherwise, $G$ fixes the set of maximal weight atoms of the measure $\lambda$ which consists either of 1 or of 2 points.

Conversely, if $G$ is compact, then it is obviously amenable. Otherwise, if $G$ fixes a point $\gamma \in \partial \mathcal{X}$, take a point $o \in \mathcal{X}$ and put $\theta_{n}=\lambda_{n}(o, \gamma)$, where $\lambda_{n}$ are the maps from Theorem 1.33 Then

$$
\left\|g \theta_{n}-\theta_{n}\right\|=\left\|g \lambda_{n}(o, \gamma)-\lambda_{n}(o, \gamma)\right\|=\left\|\lambda_{n}(g o, \gamma)-\lambda_{n}(o, \gamma)\right\| \rightarrow 0,
$$

so that Reiter's condition (3.1) is satisfied. If the fixed set of $G$ consists of two points $\left\{\gamma_{1}, \gamma_{2}\right\}$, then just take $\theta_{n}=\left[\lambda_{n}\left(o, \gamma_{1}\right)+\lambda_{n}\left(o, \gamma_{2}\right)\right] / 2$.

This result is classical for the constant curvature spaces (i.e., for Kleinian groups), and, of course, should be of no surprise in the stated generality either, although I could not find it in the literature in an explicit form. For the case of a group with a finite critical exponent acting on a CAT $(-1)$ space it is proved in BM96 Corollary 2.5] (also see BS87 and AB98 for properties of amenable groups of isometries of $\mathrm{CAT}(0)$ spaces); note that the assumption of exponential boundedness of the space $\mathcal{X}$ in Theorem 3.3 is essential (see Section 3.5 for the corresponding counterexamples).

However, the main reason for giving this proof is that it contains the key geometric ingredients of the relationship between amenability and hyperbolicity, namely, boundary convergence of geodesics (essentially equivalent to Theorem 1.33) and existence of quasi-barycenter sets, as illustrated by the following diagram: 


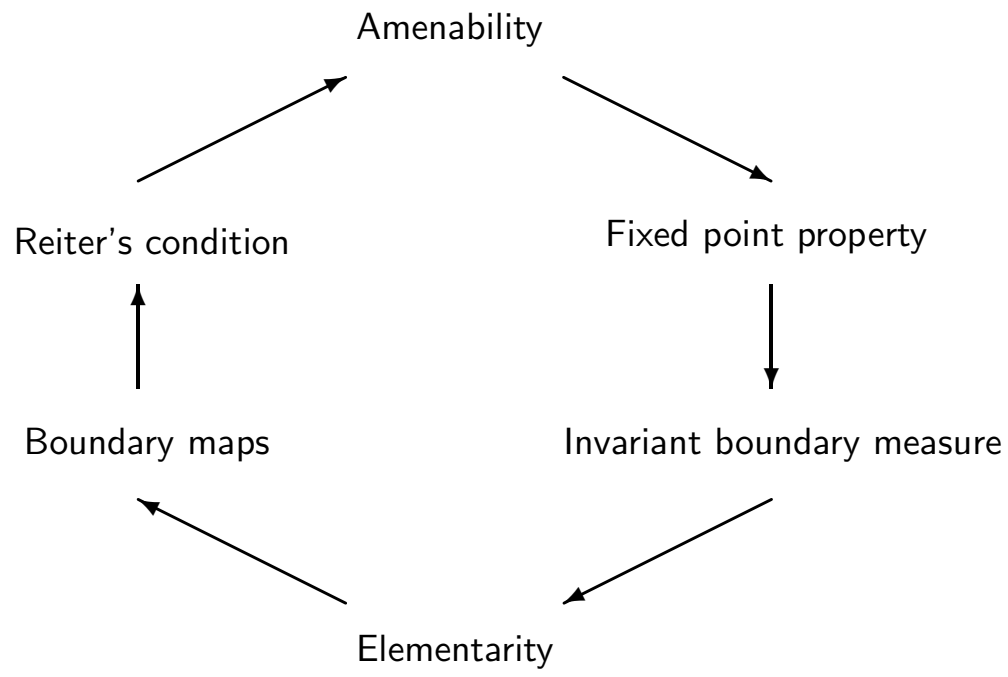

The notion of amenability can also be defined for a number of objects other than groups: discrete equivalence relations, foliations, groups actions, see [Zim78, CFW81, HK87, Ada92, AEG94, Kai97 for various definitions. All these definitions can be put into the general framework of amenability for measured groupoids, see ADR00, CHLI02. We shall refer the reader to the aforementioned references for precise definitions; note that in all these cases it is possible to give (equivalent) definitions of amenability both in terms of approximatively invariant sequences of probability measures (analogous to the Reiter condition (3.1); we shall briefly mention these definitions when dealing with the corresponding classes of objects) and in terms of an appropriate fixed point property (analogous to condition (3.2)).

We shall now proceed to establish analogues of Theorem 3.3 for these objects by taking stock of the results from Section 1 and Section 2 and following precisely the same scheme: amenability by the fixed point property implies existence of an invariant system of boundary measures, and therefore elementarity; whereas elementarity by virtue of Theorem 1.33 leads to existence of an approximatively invariant sequence of probability measures, i.e., to amenability.

\subsection{Equivalence relations.}

THEOREM 3.4. A conservative hyperbolic equivalence relation with uniformly bounded vertex degrees is amenable if and only if it is elementary.

Proof. If the equivalence relation is amenable, then the fixed point property applied to the Banach bundle of leafwise boundary measures implies existence of an invariant measurable system of boundary measures, see [ADR00 Theorem 4.2.7] (based on the original argument from [Zim77]), which by Theorem 2.20 implies that the equivalence relation is elementary.

For proving the converse implication we have to construct a sequence of measurable maps $\lambda_{n}$ assigning to a.e. point $x$ from the state space of the equivalence relation a probability measure on the equivalence class $[x]$, and such that for a.e. 
pair of equivalent points $(x, y)$

$$
\left\|\lambda_{n}(x)-\lambda_{n}(y)\right\| \rightarrow 0
$$

(this is the analogue of the Reiter condition for equivalence relations).

The only point which needs an explanation here is the measurability of the approximatively invariant sequences of measures obtained in Theorem 1.33 It would follow from the existence of a measurable family of leafwise tempered measures. Under the assumption of uniform boundedness of vertex degrees the leafwise counting measures are obviously tempered.

REMARK 3.6. S. Adams Ada90 proved that a treed equivalence relation with a finite invariant measure is amenable if and only if a.e. tree has at most 2 ends. It is plausible that the same is true for hyperbolic equivalence relations as well, namely, that for a hyperbolic equivalence relation with a finite invariant measure the hyperbolic boundary of a.e. leaf has at most 2 points.

REMARK 3.7. It is well-known that if a graphed equivalence relation has subexponential growth (i.e., $[\operatorname{card} B(x, n)]^{1 / n} \rightarrow 1$ for a.e. $x \in X$, where $B(x, n)$ are the balls in the leafwise graph metrics), then it is amenable, e.g., see AL91 Proposition 3.3]. Since the sequences of leafwise balls centered at any two equivalent points are obviously sandwiched, Lemma 1.35 provides a direct "constructive" way of proving this fact, and this Lemma can also be used in numerous other arguments deducing amenability from subexponentiality in various contexts, see AVS57, Gre69, Ser79, Sam79, ADR00, dlHGC99. However, in Theorem 1.33 Lemma 1.35 is used in a somewhat different situation, where the sandwiched sequences of sets are not metric balls.

REMARK 3.8. The boundary maps provided by Theorem 1.33 do not depend on the measure on the state space of the equivalence relation, so that in fact the argument in the proof of Theorem 3.4 shows amenability of elementary hyperbolic equivalence relations even in the Borel (rather than measure) category (cf. the discussion of definitions of amenable action in various categories in Section 3.4).

3.3. Foliations. In view of the discussion in Section 2.6 measurable foliations can be dealt with in precisely the same way as equivalence relations (by using Theorem 1.33 and Theorem 2.31). Once again, one of equivalent definitions of amenability amounts to existence of an approximatively invariant sequences of measures (3.5) (note that there is also a somewhat different notion of amenable foliation meaning that its fundamental groupoid is amenable, see the instructive discussion in CHLI02).

THEOREM 3.9. A uniform conservative hyperbolic foliation is amenable if and only if it is elementary.

COROLlary 3.10. A hyperbolic foliation admitting a compact underlying space is amenable if and only if it is elementary.

EXAMPLE 3.11. Let $\mathcal{F}$ be a foliation with simply connected leaves of pinched negative sectional curvature. Denote by $S \mathcal{F}$ the associated stable extension. This is the foliation whose underlying space consists of leafwise unit tangent vectors of $\mathcal{F}$, and the leaves consist of tangent vectors from the same leaf of $\mathcal{F}$ pointing at the same point at infinity. Then the foliation $S \mathcal{F}$ is obviously elementary, so 
that it is amenable with respect to any quasi-invariant transverse measure. This result is a generalization of the well-known amenability of the stable foliation of the geodesic flow, see Bow77. Yet another example of elementary hyperbolic foliations is provided by foliations and laminations with pointed at infinity leaves arising in conformal dynamics KL01.

REMARK 3.12. The construction of the stable extension can also be applied to an arbitrary hyperbolic foliation (not necessarily with Cartan-Hadamard leaves), in which case we shall call it the boundary extension. The underlying space of the boundary extension consists of the pairs $(x, \gamma)$, where $x$ is a point of the underlying space of the original foliation, and $\gamma$ is a point of the hyperbolic boundary of the leaf of $x$. The leaves of the boundary extension consist of all pairs $(x, \gamma)$ with the same $\gamma$. The boundary extension is elementary and therefore amenable.

3.4. Group actions. Let $G$ be a locally compact group with a measure type preserving action on a measure space $(X, \mu)$. The "Reiter type" definition of amenability of this action requires existence of a sequence of measurable maps $\lambda_{n}: X \rightarrow \mathcal{P}(G)$ which are approximatively equivariant in the sense that

$$
\left\|\lambda_{n}(g x)-g \lambda_{n}(x)\right\| \rightarrow 0
$$

for all $g \in G$ and $\mu$-a.e. $x \in X$. There are several modifications of this definition. In particular, a Borel action of a locally compact group $G$ on a Borel space $X$ is called universally amenable Ada96 (other terms: measurewise amenable ADR00, measure-amenable [JKL02) if it is amenable for any quasi-invariant measure $\mu$ on $X$. If, moreover, $X$ is a topological space, and the action of $G$ on $X$ is continuous, then the action is called topologically amenable if the convergence in (3.13) is uniform on compact subsets of $G \times X$.

THEOREM 3.14. An ergodic action of a word hyperbolic group $G$ on a measure space $(X, \mu)$ is amenable if and only if it factorizes through the action of $G$ either on the hyperbolic boundary $\partial G$, or on its symmetric square $\widetilde{\partial^{2} G}$.

Proof. The amenability of the action of $G$ on $\partial G$ or on $\widetilde{\partial^{2} G}$ is well-known (see Remark 3.16 below) and follows at once from Theorem 1.33. Therefore, any action which factorizes through $\partial G$ or $\widetilde{\partial^{2} G}$ is also amenable.

Conversely, amenability of the action on the space $(X, \mu)$ by the fixed point property applied to the Banach bundle of boundary measures implies existence of an equivariant measurable map from $X$ to the space of probability measures on $\partial G$ (in precisely the same way as in the proof of Theorem 3.4). By ergodicity we may assume that either these measures are a.e. purely non-atomic, or that almost all of them are equidistributed on a set of atoms whose cardinality is a.e. the same. In the first case, or if the number of atoms exceeds 2, we obtain by Proposition 1.18 an equivariant map from $X$ to the space of finite subsets of $G$, which means that the action on $X$ is dissipative, and therefore is confined to a single orbit. Otherwise we have the sought for equivariant projection from $X$ to $\partial G$ or $\widetilde{\partial^{2} G}$.

THEOREM 3.15. Let $G$ be a closed subgroup of the group of isometries of an exponentially bounded hyperbolic space $\mathcal{X}$. Then the action of $G$ on the hyperbolic boundary $\partial \mathcal{X}$ is topologically amenable. 
Proof. A sequence of approximatively invariant measures for this action is provided by Theorem 1.33. Indeed, let $\lambda_{n}: \mathcal{X} \times \partial \mathcal{X} \rightarrow \mathcal{P}(\mathcal{X})$ be the $\operatorname{Iso}(\mathcal{X})$ equivariant maps constructed in Theorem 1.33. Choose a reference point $o \in \mathcal{X}$, and put $\widetilde{\lambda}_{n}(\gamma)=\lambda_{n}(o, \gamma)$ for any $g \in G$ and $\gamma \in \partial \mathcal{X}$. Then the maps $\widetilde{\lambda}_{n}: \partial \mathcal{X} \rightarrow \mathcal{P}(\mathcal{X})$ are approximatively equivariant, because

$$
\left\|\widetilde{\lambda}_{n}(g \gamma)-g \widetilde{\lambda}_{n}(\gamma)\right\|=\left\|\lambda_{n}(o, g \gamma)-\lambda_{n}(g o, g \gamma)\right\| \rightarrow 0
$$

for any $g \in G$ and $\gamma \in \partial \mathcal{X}$ uniformly on compacts, see Remark 1.41 Since the action of $G$ on $\mathcal{X}$ is proper, there exists an equivariant Borel lift $\mathcal{P}(\mathcal{X}) \rightarrow \mathcal{P}(G)$ (e.g., see [ADR00 Proposition 2.1.10]). Applying it to the sequence $\widetilde{\lambda}_{n}$ gives an approximatively equivariant sequence of maps from $\partial \mathcal{X}$ to $\mathcal{P}(G)$.

REMARK 3.16. The action of the group $S L(2, \mathbb{Z})$ on the circle at infinity of the hyperbolic plane was the first example of an amenable action of a non-amenable group Bow77, Ver78. Since then amenability of boundary actions was established in a number of other situations Spa87, SZ91, Ada94; the most general result being universal amenability of the boundary action of the group of isometries of an exponentially bounded hyperbolic space Ada96. In the particular case of the boundary action of a word hyperbolic group the arguments of Adams were recently simplified by Germain Ger00, who proved topological amenability of this action. However, the approach based on using Theorem 1.33 and Lemma 1.35 is more streamlined even in this case.

In the CAT $(-1)$ case replacing Theorem 1.33 with Theorem 1.38 in the proof of Theorem 3.15 yields

THEOREM 3.17. Let $G$ be a closed subgroup of the group of isometries of a hyperbolic space $\mathcal{X}$ with finite critical exponent. Then the action of $G$ on the hyperbolic boundary $\partial \mathcal{X}$ is topologically amenable.

Remark 3.18. Burger and Mozes in BM96 Corollary 1.4] stopped short of saying that under the assumptions of Theorem 3.17 the action of $G$ on $\partial \mathcal{X}$ is amenable with respect to any Patterson measure (although they basically proved that). Moreover, they also made the first step to establishing universal amenability of the boundary action by showing that the stabilizer of any boundary point is amenable (which is a necessary condition for universal amenability of the action, see Ada92).

Remark 3.19. As we shall see in Section 3.5 below, without any assumptions on the growth of the space $\mathcal{X}$ Theorem [3.15] and Theorem 3.17 may fail even in the weakest form of amenability of the action with respect to a given quasi-invariant boundary measure, which answers a question formulated by Adams in Ada96.

3.5. Counterexamples to boundary amenability. As it was pointed out by Burger and Mozes in [BM96] (Remark after Proposition 1.6; in our notations $X(\infty) \equiv \partial X)$,

The product $X=[0, \infty) \times \mathbf{H}^{2}$ with Riemannian metric $(d t)^{2}+e^{-2 t} \frac{|d z|^{2}}{y^{2}}$, where $\mathbf{H}^{2}$ denotes the upper half-plane, is a $\operatorname{CAT}(-1)$ space on which $S L(2, \mathbb{R})$ acts isometrically, fixing a point in $X(\infty)$.

Note that the metric on $X$ is obtained from the hyperbolic metric on $\mathbf{H}^{2}$ in precisely the same way as the hyperbolic metric on (a horoball in) $\mathbf{H}^{2}$ is obtained from 
the Euclidean metric on $\mathbb{R}$, i.e., by exponential rescaling in the vertical direction. In fact, this example is a particular case of the following general hyperbolization construction (cf. KL01]). This construction resembles that of a $\kappa$-cone over a metric space (e.g., see [BH99]).

Proposition 3.20. For a complete $\mathrm{CAT}(0)$ space $Y$ put $X=\mathbb{R} \times Y$, and define the metric $d_{X}$ on $X$ by putting for any two points $\left(t_{1}, y_{1}\right),\left(t_{2}, y_{2}\right) \in X$

$$
d_{X}\left(\left(t_{1}, y_{1}\right),\left(t_{2}, y_{2}\right)\right)=d_{\mathbf{H}^{2}}\left(\left(t_{1}, 0\right),\left(t_{2}, d_{Y}\left(y_{1}, y_{2}\right)\right)\right)
$$

where $d_{Y}$ is the metric on $Y$, and $d_{\mathbf{H}^{2}}$ is the metric

$$
d s^{2}=d t^{2}+e^{-2 t} d l^{2}
$$

on the hyperbolic plane $\mathbf{H}^{2} \cong \mathbb{R} \times \mathbb{R}$ realized in the upper half-plane model with logarithmic rescaling of the vertical coordinate. Then $d_{X}$ is a complete metric on $X$, the space $X$ is $\mathrm{CAT}(-1)$ with respect to this metric, and the boundary $\partial X$ of the hyperbolic compactification of $X$ is the union $Y \cup\{\omega\}$, where $\omega$ is the common limit point of vertical geodesic rays $[0, \infty) \times\{y\}, y \in Y$ in $X$ directed upwards. The point of $\partial X$ corresponding to a point $y \in Y$ is the limit point of the directed downwards vertical geodesic ray $[0,-\infty) \times\{y\}$. If $Y$ is non-compact, then $\partial X$ is homeomorphic to the one-point compactification of $Y$, and if $Y$ is compact, then $\partial Y$ is homeomorphic to the disjoint union of $Y$ and $\omega$.

Definition 3.22. We shall call the $\operatorname{CAT}(-1)$ space $\left(X, d_{X}\right)=\left(\mathcal{H} Y, d_{\mathcal{H} Y}\right)$ constructed in Proposition 3.20 the hyperbolization of the $\operatorname{CAT}(0)$ space $\left(Y, d_{Y}\right)$.

Proof of Proposition 3.20] The verification of the triangle inequality for $d_{X}$ using formula (3.21) is straightforward. Completeness of the metric $d_{X}$ is obvious. Further, the space $\left(X, d_{X}\right)$ is geodesic, and the (unique) geodesic $\bar{\xi}$ joining two points $\left(t_{1}, y_{1}\right)$ and $\left(t_{2}, y_{2}\right)$ is the image of the geodesic joining the points $\left(t_{1}, 0\right)$ and $\left(t_{2}, d_{Y}\left(y_{1}, y_{2}\right)\right)$ in $\mathbf{H}^{2}$ under the natural isometry between the strip $\mathbb{R} \times\left[0, d_{y}\left(y_{1}, y_{2}\right)\right]$ in $\mathbf{H}^{2}$ and the "curtain" $\mathbb{R} \times \xi$ in $X$ over the geodesic $\xi$ joining $y_{1}$ and $y_{2}$ in $Y$.

In order to check the $\mathrm{CAT}(-1)$ property let us consider a geodesic triangle $\Delta_{X}=\left[\left(t_{0}, y_{0}\right),\left(t_{1}, y_{1}\right),\left(t_{2}, y_{2}\right)\right]$ in $X$. It is more convenient to construct a comparison triangle for $\Delta_{X}$ in the 3-dimensional hyperbolic space $\mathbf{H}^{3}$ rather than in the 2-dimensional hyperbolic plane $\mathbf{H}^{2}$, and to use the fact that $\mathbf{H}^{3}$ is obtained from the Euclidean plane $\mathbf{E}^{2}$ in precisely the same way as $X$ is obtained from $Y$. Let $\Delta_{\mathbf{E}^{2}}=\left[z_{0}, z_{1}, z_{2}\right]$ be the comparison triangle in $\mathbf{E}^{2}$ for the triangle $\Delta_{Y}=\left[y_{0}, y_{1}, y_{2}\right]$ in $Y$. Then the definition of the distance $d_{X}$ implies that $\Delta_{\mathbf{H}^{3}}=\left[\left(t_{0}, z_{0}\right),\left(t_{1}, z_{1}\right),\left(t_{2}, z_{2}\right)\right]$ is a comparison triangle for $\Delta_{X}$. Now take points $\left(t_{i}^{\prime}, y_{i}^{\prime}\right), i=1,2$, on the sides $\left[\left(t_{0}, y_{0}\right),\left(t_{i}, y_{i}\right)\right]$ of the triangle $\Delta_{X}$, and denote by $\left(t_{i}^{\prime}, z_{i}^{\prime}\right)$ the corresponding points on the sides of the comparison triangle $\Delta_{\mathbf{H}^{3}}$. By the above description of the geodesics in $X$ the "heights" $t_{i}^{\prime}$ remain the same, and $d_{Y}\left(y_{0}, y_{i}^{\prime}\right)=d_{\mathbf{E}^{2}}\left(z_{0}, z_{i}^{\prime}\right)$. Therefore the CAT(0) property of the space $Y$ and the definition of the metric $d_{X}$ imply that

$$
d_{X}\left(\left(t_{1}^{\prime}, y_{1}^{\prime}\right),\left(t_{2}^{\prime}, y_{2}^{\prime}\right)\right) \leq d_{\mathbf{H}^{3}}\left(\left(t_{1}^{\prime}, z_{1}^{\prime}\right),\left(t_{2}^{\prime}, z_{2}^{\prime}\right)\right) .
$$

The description of the hyperbolic boundary $\partial X$ easily follows from the above description of the geodesics in $X$.

Proposition 3.20 now allows one to obtain examples showing that Theorem 3.3. Theorem 3.15] and Theorem 3.17 may well fail without any bounded geometry assumptions on the space or on the group. 
Proposition 3.23. Let $Y$ be any $\mathrm{CAT}(0)$ space with a non-amenable closed group of isometries $G$, and let $X=\mathcal{H} Y$ be its hyperbolization as described in Proposition 3.20. Then the group $G$ acting on $X$ as $g(t, y)=(t, g y)$ is also closed in $\operatorname{Iso}(X)$, and it fixes the point $\omega \in \partial X$. Therefore,

- The group $G$ is elementary with respect to the space $X$ and non-amenable;

- The action of the group $G$ on $\partial X$ is non-amenable with respect to the invariant measure $\delta_{\omega}$ concentrated at the fixed point $\omega$.

In order to obtain more concrete examples one can take $Y$ to be, for instance, the hyperbolic plane (as did Burger and Mozes; actually they took just the horoball in $\mathcal{H} \mathbf{H}^{2}$ centered at the point $\omega$ rather than the whole space $\mathcal{H} \mathbf{H}^{2}$ ), or the Cayley 1-complex of the free group. Still, it would be interesting to have examples of non-amenable actions with respect to a non-atomic boundary measure.

\section{References}

[AB98] Scot Adams and Werner Ballmann, Amenable isometry groups of Hadamard spaces, Math. Ann. 312 (1998), no. 1, 183-195. MR 99i:53032

[Ada90] Scot Adams, Trees and amenable equivalence relations, Ergodic Theory Dynam. Systems 10 (1990), no. 1, 1-14. MR 91d:28041

[Ada92] _ Generalities on amenable actions, Unpublished notes.

[Ada94] B Boundary amenability for word hyperbolic groups and an application to smooth dynamics of simple groups, Topology 33 (1994), no. 4, 765-783. MR 96g:58104

[Ada96] Reduction of cocycles with hyperbolic targets, Ergodic Theory Dynam. Systems 16 (1996), no. 6, 1111-1145. MR 98i:58135

[ADR00] C. Anantharaman-Delaroche and J. Renault, Amenable groupoids, Monographies de L'Enseignement Mathématique [Monographs of L'Enseignement Mathématique], vol. 36, L'Enseignement Mathématique, Geneva, 2000, With a foreword by Georges Skandalis and Appendix B by E. Germain. MR 2001m:22005

[AEG94] Scot Adams, George A. Elliott, and Thierry Giordano, Amenable actions of groups, Trans. Amer. Math. Soc. 344 (1994), no. 2, 803-822. MR 94k:22010

[AL91] Scot Adams and Russell Lyons, Amenability, Kazhdan's property and percolation for trees, groups and equivalence relations, Israel J. Math. 75 (1991), no. 2-3, 341-370. MR 93j: 43001

[AVS57] G. M. Adelson-Velskii and Yu. A. Shreider, The Banach mean on groups, Uspehi Mat. Nauk (N.S.) 12 (1957), no. 6(78), 131-136. MR 20 \#1238

[BCG96] Gérard Besson, Gilles Courtois, and Sylvestre Gallot, Minimal entropy and Mostow's rigidity theorems, Ergodic Theory Dynam. Systems 16 (1996), no. 4, 623-649. MR 97e:58177

[BH99] Martin R. Bridson and André Haefliger, Metric spaces of non-positive curvature, Grundlehren der Mathematischen Wissenschaften [Fundamental Principles of Mathematical Sciences], vol. 319, Springer-Verlag, Berlin, 1999. MR 2000k:53038

[BM96] M. Burger and S. Mozes, CAT(-1)-spaces, divergence groups and their commensurators, J. Amer. Math. Soc. 9 (1996), no. 1, 57-93. MR 96c:20065

[Bow77] Rufus Bowen, Anosov foliations are hyperfinite, Ann. of Math. (2) 106 (1977), no. 3, 549-565. MR 57 \#1569

[BS87] M. Burger and V. Schroeder, Amenable groups and stabilizers of measures on the boundary of a Hadamard manifold, Math. Ann. 276 (1987), no. 3, 505-514. MR 88b:53049

[CFW81] A. Connes, J. Feldman, and B. Weiss, An amenable equivalence relation is generated by a single transformation, Ergodic Theory Dynamical Systems 1 (1981), no. 4, 431450 (1982). MR 84h:46090

[CHLI02] K. Corlette, L. Hernández Lamoneda, and A. Iozzi, A vanishing theorem for the tangential de Rham cohomology of a foliation with amenable fundamental groupoid, Preprint. 
[DE86] Adrien Douady and Clifford J. Earle, Conformally natural extension of homeomorphisms of the circle, Acta Math. 157 (1986), no. 1-2, 23-48. MR 87j:30041

[dlHGC99] P. de la Harpe, R. I. Grigorchuk, and T. Ceccherini, Amenability and paradoxical decompositions for pseudogroups and discrete metric spaces, Tr. Mat. Inst. Steklova 224 (1999), 68-111. MR 2001h:43001

[FD88] J. M. G. Fell and R. S. Doran, Representations of* -algebras, locally compact groups, and Banach *-algebraic bundles. Vol. 1, Pure and Applied Mathematics, vol. 125, Academic Press Inc., Boston, MA, 1988, Basic representation theory of groups and algebras. MR 90c: 46001

[FM77] Jacob Feldman and Calvin C. Moore, Ergodic equivalence relations, cohomology, and von Neumann algebras. I, Trans. Amer. Math. Soc. 234 (1977), no. 2, 289-324. MR 58 \#28261a

[GdlH90] É. Ghys and P. de la Harpe (eds.), Sur les groupes hyperboliques d'après Mikhael Gromov, Progress in Mathematics, vol. 83, Birkhäuser Boston Inc., Boston, MA, 1990, Papers from the Swiss Seminar on Hyperbolic Groups held in Bern, 1988. MR 92f: 53050

[Ger00] E. Germain, Approximate invariant means for boundary actions of hyperbolic groups, 2000, Appendix B in the book ADR00.

[Gre69] Frederick P. Greenleaf, Invariant means on topological groups and their applications, Van Nostrand Mathematical Studies, No. 16, Van Nostrand Reinhold Co., New York, 1969. MR 40 \#4776

[Gro87] M. Gromov, Hyperbolic groups, Essays in group theory, Math. Sci. Res. Inst. Publ., vol. 8, Springer, New York, 1987, pp. 75-263. MR 89e:20070

[HK87] S. Hurder and A. Katok, Ergodic theory and Weil measures for foliations, Ann. of Math. (2) 126 (1987), no. 2, 221-275. MR 89d:57042

[JKL02] S. Jackson, A. S. Kechris, and A. Louveau, Countable Borel equivalence relations, J. Math. Log. 2 (2002), no. 1, 1-80. MR 2003f:03066

[Kai94] Vadim A. Kaimanovich, Ergodicity of harmonic invariant measures for the geodesic flow on hyperbolic spaces, J. Reine Angew. Math. 455 (1994), 57-103. MR 95g:58130

[Kai97] _ Amenability, hyperfiniteness, and isoperimetric inequalities, C. R. Acad. Sci. Paris Sér. I Math. 325 (1997), no. 9, 999-1004. MR 98j:28014

[Kai02] _ Random walks on Sierpinski graphs: hyperbolicity and stochastic homogenization, Preprint.

[KL01] Vadim A. Kaimanovich and Mikhail Lyubich, Conformal and harmonic measures on laminations associated with rational maps, 2001, Preprint.

[MS88] Calvin C. Moore and Claude Schochet, Global analysis on foliated spaces, Mathematical Sciences Research Institute Publications, vol. 9, Springer-Verlag, New York, 1988, With appendices by S. Hurder, Moore, Schochet and Robert J. Zimmer. MR 89h:58184

[Pat76] S. J. Patterson, The limit set of a Fuchsian group, Acta Math. 136 (1976), no. 3-4, 241-273. MR 56 \#8841

[Pat88] Alan L. T. Paterson, Amenability, Mathematical Surveys and Monographs, vol. 29, American Mathematical Society, Providence, RI, 1988. MR 90e:43001

[Pie84] Jean-Paul Pier, Amenable locally compact groups, Pure and Applied Mathematics, John Wiley \& Sons Inc., New York, 1984, A Wiley-Interscience Publication. MR 86a:43001

[Pla75] J. F. Plante, Foliations with measure preserving holonomy, Ann. of Math. (2) 102 (1975), no. 2, 327-361. MR 52 \#11947

[Sam79] Manuel Samuélidès, Tout feuilletage à croissance polynomiale est hyperfini, J. Funct. Anal. 34 (1979), no. 3, 363-369. MR 81 m:57021

[Ser79] Caroline Series, Foliations of polynomial growth are hyperfinite, Israel J. Math. 34 (1979), no. 3, 245-258 (1980). MR 82i:28019

[Spa87] R. J. Spatzier, An example of an amenable action from geometry, Ergodic Theory Dynam. Systems 7 (1987), no. 2, 289-293. MR 88j:58100

[SZ91] Ralf J. Spatzier and Robert J. Zimmer, Fundamental groups of negatively curved manifolds and actions of semisimple groups, Topology 30 (1991), no. 4, 591-601. MR 92m:57047 
[Ver78] A. M. Vershik, The action of $\operatorname{PSL}(2, \mathbf{Z})$ in $\mathbf{R}^{1}$ is approximable, Uspehi Mat. Nauk 33 (1978), no. 1(199), 209-210. MR 58 \#13201

[Woe00] Wolfgang Woess, Random walks on infinite graphs and groups, Cambridge Tracts in Mathematics, vol. 138, Cambridge University Press, Cambridge, 2000. MR 2001k:60006

[Zim77] Robert J. Zimmer, Hyperfinite factors and amenable ergodic actions, Invent. Math. 41 (1977), no. 1, 23-31. MR 57 \#10438

[Zim78] - Amenable ergodic group actions and an application to Poisson boundaries of random walks, J. Functional Analysis 27 (1978), no. 3, 350-372. MR 57 \#12775

CNRS UmR 6625, IRMaR, Université Rennes-1, Campus Beaulieu, 35042 Rennes, FRANCE

E-mail address: kaimanov@univ-rennes1.fr 